hep-th/9912181

\title{
Duality in Heterotic Vacua With Four Supercharges@
}

\author{
Michael Haackf and Jan Louis? \\ Fachbereich Physik, Martin-Luther-Universiät Halle-Wittenberg, \\ Friedemann-Bach-Platz 6, D-06099 Halle, Germany
}

\begin{abstract}
We study heterotic vacua with four supercharges in three and four space-time dimensions and their duals obtained as M/F-theory compactified on Calabi-Yau fourfolds. We focus on their respective moduli spaces and derive the Kähler potential for heterotic vacua obtained as circle compactifications of four-dimensional $N=1$ heterotic theories. The Kähler potential of the dual theory is computed by compactifying 11-dimensional supergravity on Calabi-Yau fourfolds. The duality between these theories is checked for K3-fibred fourfolds and an appropriate F-theory limit is discussed.
\end{abstract}

December 1999

\footnotetext{
${ }^{1}$ Work supported by: GIF - the German-Israeli Foundation for Scientific Research, DAAD - the German Academic Exchange Service and the Landesgraduiertenförderung Sachsen-Anhalt.

${ }^{2}$ email: michael@hera1.physik.uni-halle.de

${ }^{3}$ email: j.louis@physik.uni-halle.de
} 


\section{Introduction}

The $E_{8} \times E_{8}$ heterotic string has been the prime candidate for providing a string theoretic version of the (supersymmetric) Standard Model for over a decade. In particular, the vacuum solutions with 4 Minkowskian space-time dimensions $(D=4)$ and $N=1$ supersymmetry have been extensively studied due to their phenomenological prospects. However, despite considerable efforts a number of (serious) problems remain within the framework of the perturbative heterotic string such as a missing mechanism for hierarchical supersymmetry breaking and the stabilization of the dilaton.

With the advent of string dualities two things have changed. On the one hand it has been possible to control some of the non-perturbative properties (or couplings) of string theory [1]. On the other hand the $S O(32)$ heterotic string and even more so the type I string have been investigated from the point of view of viable particle phenomenology [2].

In this paper we continue to focus on $N=1$ vacua of the heterotic string but include their circle compactifications to $D=3$ into our considerations. The reason is that in $D=3$ the vector multiplet contains a real scalar in the adjoint representation of the heterotic gauge group and thus - contrary to the situation in $D=4$ - a Coulomb branch exists. Some of the non-perturbative features of these vacua are believed to be captured by F-theory compactified on elliptic Calabi-Yau fourfolds $Y_{4}$ or M-theory compactified on $Y_{4}$ for the three-dimensional case 3 , 41$]$.

The purpose of this paper is to facilitate a comparison of the corresponding effective Lagrangians and in particular the identification of those $\mathrm{M} / \mathrm{F}$-theory vacua which have a perturbative heterotic limit. In our analysis we ignore all charged matter multiplets and only focus on the moduli which are constrained by supersymmetry to parameterize a Kähler manifold [42, 43]. We derive the Kähler potential for the moduli of a CalabiYau fourfold in the large volume limit of M-theory including the moduli coming from the three-form potential and compare it to the Kähler potential of the corresponding heterotic vacua. In spirit our analysis is very close to a similar analysis carried out for the duality of type IIA on a Calabi-Yau threefold $Y_{3}$ and the heterotic string on $\mathrm{K} 3 \times T^{2}$ in refs. 44, 45.

The organisation of the paper is as follows. In section 2 (and appendix B) we start with a general effective supergravity Lagrangian in $N=1, D=4$ which can be obtained by compactifications of the heterotic string on an appropriate $(0,2)$ superconformal field theory and perform a Kaluza-Klein reduction to $D=3$ on a circle. We derive the threedimensional Kähler potential in terms of the four-dimensional one and an additional term coming from the four-dimensional vector fields. In section 3 (and appendix C) we compactify 11-dimensional supergravity on a Calabi-Yau fourfold with vanishing Euler number $(\chi=0)$ and vanishing four-form flux. We derive the Kähler potential for the moduli which include the geometrical moduli arising as deformations of the Kähler class and complex structure of the fourfold as well as the moduli coming from the expansion of the M-theory three-form. The M-theory Kähler potential is compared to the heterotic Kähler potential in section 1 . We show that for fourfolds which are K3-fibrations over a complex two-dimensional base $B_{2}$ the two Kähler potentials agree in the limit of large

\footnotetext{
${ }^{4}$ This includes in particular compactifications on Calabi-Yau threefolds $Y_{3}$ with a choice of the gauge bundle.
} 
$B_{2}$ and weak heterotic coupling. Finally, the F-theory limit is discussed.

Section 5 contains our conclusions and appendix A assembles notation and conventions. A preliminary version of some of our results was given in 46.

\section{Heterotic String Vacua}

\section{1 $D=4, N=1$ heterotic vacua}

Our starting point is a generic effective supergravity Lagrangian in $D=4$ with $N=1$ supersymmetry. Such vacua can be constructed as compactifications of the heterotic string on Calabi-Yau threefolds $Y_{3}$ or more generally from appropriate $(0,2)$ superconformal field theories. For the purpose of this paper it is sufficient to focus only on the vector multiplets and the chiral moduli multiplets and ignore all charged matter multiplets. The effective Lagrangian in this case reads [47]

$$
\begin{aligned}
\mathcal{L}^{(4)}= & \frac{1}{2} R^{(4)}-G_{\bar{I} J}^{(4)}(\Phi, \bar{\Phi}) \partial_{m} \bar{\Phi}^{\bar{I}} \partial^{m} \Phi^{J}-\frac{1}{4} \operatorname{Re} f_{a b}(\Phi) F_{m n}^{a} F^{b m n}+\frac{1}{4} \operatorname{Im} f_{a b}(\Phi) F_{m n}^{a} \tilde{F}^{b m n} \\
& +\ldots,
\end{aligned}
$$

where $m, n=0, \ldots, 3, \Phi^{I}$ are the moduli fields and $F_{m n}^{a}$ is the field strength of the gauge bosons $A_{m}^{a}$. The index a labels the generators of the gauge group $G$ and thus $a=1, \ldots, \operatorname{dim}(G) . \quad N=1$ supersymmetry requires that the $f_{a b}(\Phi)$ are holomorphic functions of the moduli which are further constrained by gauge invariance. The metric $G_{\bar{I} J}^{(4)}$ has to be a Kähler metric, that is

$$
G_{\bar{I} J}^{(4)}=\bar{\partial}_{\bar{I}} \partial_{J} K^{(4)}(\Phi, \bar{\Phi})
$$

where $K^{(4)}$ is the Kähler potential.

Perturbative heterotic string theory imposes further constraints on the functions $K^{(4)}$ and $f_{a b}$. First of all the rank $\mathrm{r}$ of the gauge group $G$ is bounded by the central charge $c$ of the left moving (bosonic) conformal field theory on the worldsheet. For heterotic strings in $D=4$ one has $c=22$ and hence

$$
\mathrm{r}(G) \leq 22
$$

Secondly, the holomorphic $f_{a b}$ are universal at the string tree level and determined by the heterotic dilaton $\lambda_{4}$ and the axion $a$ which is the (Poincaré) dual of the antisymmetric tensor $B_{m n}$. The two scalars are combined into a complex $S=\lambda_{4}^{-2}+i a$ and one has

$$
f_{a b}=k_{a} S \delta_{a b}+\ldots
$$

where $k$ is the level of the Kac-Moody algebra. The ... denote perturbative and nonperturbative quantum corrections which are suppressed in the large $S$ (weak coupling) limit; they play no role in this paper.

\footnotetext{
${ }^{5}$ We neglect the possibility of anomalous $U(1)$ gauge factors with appropriate four-dimensional GreenSchwarz terms.
} 
The metric of the moduli also simplifies at the string tree level. Using the notation $\Phi^{I}=\left(S, \phi^{i}\right)$ with $i=1, \ldots, n_{4}, I=0, \ldots, n_{4}$ one has

$$
K^{(4)}=-\ln (S+\bar{S})+\tilde{K}^{(4)}(\phi, \bar{\phi})+\ldots,
$$

where $\tilde{K}^{(4)}(\phi, \bar{\phi})$ is the tree level Kähler potential for all moduli but the dilaton. It is a model dependent function and does not enjoy any generic properties. For Calabi-Yau compactifications with the standard embedding of the spin connection (so called $(2,2)$ vacua) $\tilde{K}^{(4)}$ splits into a sum

$$
\tilde{K}^{(4)}=K_{1,1}^{(4)}+K_{2,1}^{(4)}
$$

where $K_{1,1}^{(4)}\left(K_{2,1}^{(4)}\right)$ is the Kähler potential for the $(1,1)$-moduli $\left((2,1)\right.$-moduli) of $Y_{3}$. For future reference we also need to recall that in the large volume limit of Calabi-Yau compactifications $K_{1,1}^{(4)}$ is given by 48,49.

$$
K_{1,1}^{(4)}=-\ln \left[d_{A B C}(t+\bar{t})^{A}(t+\bar{t})^{B}(t+\bar{t})^{C}\right]=-\ln \left[\operatorname{Vol}\left(Y_{3}\right)\right]
$$

where $d_{A B C}$ are the (classical) intersection numbers, $t^{A}$ the $(1,1)$-moduli and $\operatorname{Vol}\left(Y_{3}\right)$ is the classical volume of the compactification manifold $Y_{3}$. For $K_{2,1}^{(4)}$ one has instead 49, 50

$$
K_{2,1}^{(4)}=-\ln \left[\int_{Y_{3}} \Omega \wedge \bar{\Omega}\right]
$$

where $\Omega$ is the unique $(3,0)$-form.

Finally, let us note that the couplings of the dilaton in eqs. (4) and (5) are largely determined by the fact that $\lambda_{4}$ organizes the string perturbation theory and that in perturbation theory there is a continuous Peccei-Quinn (PQ) symmetry $S \rightarrow S+i \gamma, \gamma \in \mathbb{R}$ shifting the axion $a$.

\section{2 $D=4, N=1$ supergravity compactified on $S^{1}$}

Let us reduce the Lagrangian (1) to $D=3$ on a circle $S^{1}$. This does not break any supercharges so that the theory continues to have 4 real supercharges. In $D=3$ this corresponds to $N=2$ supersymmetry since the irreducible Majorana spinor has 2 real components.

For the $S^{1}$-reduction to $D=3$ we use the Ansatz [52]:

$$
g_{m n}^{(4)}=\left(\begin{array}{cc}
g_{\mu \nu}^{(3)}+r^{2} B_{\mu} B_{\nu} & r^{2} B_{\mu} \\
r^{2} B_{\nu} & r^{2}
\end{array}\right), \quad A_{m}^{a}=\left(A_{\mu}^{a}+B_{\mu} \zeta^{a}, \zeta^{a}\right)
$$

where $\mu, \nu=0,1,2$ and $r$ is the radius (measured in the $D=4$ Einstein metric) of the $S^{1}$. The reduction procedure follows closely ref. [52] where four-dimensional $N=2$ vacua are considered. The details for the reduction of $N=1$ vacua are shown in appendix $\mathbb{B}$ and here we only give the results.

In $D=3$ the vector multiplets contain the real scalars $\zeta^{a}$ in the adjoint representation of $G$. Thus there is an additional component of the moduli space (a Coulomb branch)

\footnotetext{
${ }^{6} K_{2,1}^{(4)}$ has a similar expansion as $K_{1,1}^{(4)}$ around the large complex structure point 51 .
} 
spanned by the scalar fields lying in the Cartan subalgebra of $G$ and at a generic point in this moduli space the gauge group is $[U(1)]^{\mathrm{r}(G)}$. In order to make the notation not too heavy we continue to label the $U(1)$ gauge multiplets by the index $a$ although in the previous section the same index ran over all gauge generators; thus $a=1, \ldots, \mathrm{r}(G)$ henceforth.

In $D=3$ an Abelian vector is (Poincaré) dual to a scalar and thus a vector multiplet is dual to a chiral multiplet. Technically this duality is seen by adding Lagrange multipliers $C^{a}, b$ to the three-dimensional Lagrangian and eliminating $A_{\mu}^{a}, B_{\mu}$ by their equations of motion (see appendix B). In this dual picture all supermultiplets are chiral and thus their scalar fields have to parametrize a Kähler manifold [43]. It turns out that the Kähler structure only becomes manifest after introducing the coordinates

$$
\begin{aligned}
D^{a} & \equiv-f_{a b}(\Phi) \zeta^{b}+i C^{a}, \\
T & \equiv r^{2}+i b+\frac{1}{2}(\operatorname{Re} f)_{a b}^{-1}(\Phi) D^{a}\left(D^{b}+\bar{D}^{b}\right) .
\end{aligned}
$$

Combining all $n_{4}+\mathrm{r}(G)+2$ scalar fields into the coordinate vector $Z^{\Sigma}=\left(S, \phi^{i}, D^{a}, T\right), \Sigma=$ $0, \ldots, n_{4}+\mathrm{r}(G)+1$ the three-dimensional Lagrangian can be written as

$$
\mathcal{L}^{(3)}=\frac{1}{2} R^{(3)}-G_{\bar{\Lambda} \Sigma} \partial_{\mu} \bar{Z}^{\bar{\Lambda}} \partial^{\mu} Z^{\Sigma}
$$

where $G_{\bar{\Lambda} \Sigma}$ obeys

$$
\begin{aligned}
G_{\bar{\Lambda} \Sigma} & =\bar{\partial}_{\bar{\Lambda}} \partial_{\Sigma} K_{\text {het }}^{(3)} \\
K_{\text {het }}^{(3)} & =K^{(4)}(\Phi, \bar{\Phi})-\ln \left[T+\bar{T}-\frac{1}{2}(D+\bar{D})^{a}(\operatorname{Re} f)_{a b}^{-1}(D+\bar{D})^{b}\right] .
\end{aligned}
$$

Note that the argument of the logarithm is given by the square of the compactification radius, as can be seen from eq. (10):

$$
T+\bar{T}-\frac{1}{2}(D+\bar{D})^{a}(\operatorname{Re} f)_{a b}^{-1}(D+\bar{D})^{b}=2 r^{2}
$$

\subsection{The heterotic $D=3$ low energy effective Lagrangian}

So far the reduction did not use any input from string theory but was merely an 'exercise' in supergravity. Inserting the string tree level properties displayed in eqs. (4) and (可) into $K_{\text {het }}^{(3)}$ of eq. (12) yields

$$
K_{\text {het }}^{(3)}=\tilde{K}^{(4)}(\phi, \bar{\phi})-\ln \left[(T+\bar{T})(S+\bar{S})-\left(D^{a}+\bar{D}^{a}\right)^{2}\right] .
$$

We will see in section 4 that in the dual M-theory vacua we are more naturally led to the coordinates $S^{\prime}=\frac{1}{2}(S+T)$ and $T^{\prime}=\frac{1}{2}(S-T)$ and the Kähler potential

$$
K_{\text {het }}^{(3)}=\tilde{K}^{(4)}(\phi, \bar{\phi})-\ln \left[\left(S^{\prime}+\bar{S}^{\prime}\right)^{2}-\left(T^{\prime}+\bar{T}^{\prime}\right)^{2}-\left(D^{a}+\bar{D}^{a}\right)^{2}\right] .
$$

The next step is to identify the three-dimensional dilaton. The relation to the 4-dimensional dilaton is as usually

$$
\frac{1}{\lambda_{3}^{2}}=\frac{r_{s}}{\lambda_{4}^{2}}
$$


where $r_{s}$ is the radius of $S^{1}$ measured in the four-dimensional string frame metric. In the reduction procedure we used the metric in the Einstein frame (eq. (9)) which is related

to the metric in the string frame by the Weyl rescaling $g_{\mathrm{E}}^{(4)}=\lambda_{4}^{-2} g_{\mathrm{str}}^{(4)}$. This implies the following relation among the radii

$$
r_{s}=r \lambda_{4}
$$

Combining (16) and (17) results in

$$
\lambda_{3}^{2}=\frac{\lambda_{4}}{r}
$$

Using (13) and $\lambda_{4}^{-2}=\frac{1}{2}(S+\bar{S})$ we also derive

$$
\lambda_{3}^{-4}=(T+\bar{T})(S+\bar{S})-\left(D^{a}+\bar{D}^{a}\right)^{2} .
$$

The three-dimensional dilaton governs the perturbation series in $D=3$, as can be seen by reducing the four-dimensional Lagrangian in the string frame. The result can be found in appendix B. The Kähler potential of eq. (14) is only valid to lowest order in $r$ and $\lambda_{3}$ and gets perturbative and non-perturbative corrections.

Finally let us discuss the symmetries of the compactified theory. First of all there is the PQ associated with the four-dimensional axion $a$ discussed in section 2.1. Furthermore, there are $\mathrm{r}(G)+1$ Abelian gauge symmetries associated with the $\mathrm{r}(G)+1$ gauge bosons $A_{\mu}^{a}, B_{\mu}$. In the dual Lagrangian these symmetries appear as continuous PQ symmetries acting on the dual scalars. Finally, the scalars $\zeta^{a}$ in the three-dimensional vector multiplets 'inherit' another PQ symmetry from the four-dimensional gauge invariance. So altogether (11), (12) are invariant under the following $2 \mathrm{r}(G)+2 \mathrm{PQ}$ symmetries (with parameters $\left.\gamma, \tilde{\gamma}, \gamma^{a}, \hat{\gamma}^{a}\right)$

$$
\begin{aligned}
a & \rightarrow a+\gamma, \\
b & \rightarrow b+\tilde{\gamma}, \\
C^{a} & \rightarrow C^{a}+\gamma^{a}, \quad b \rightarrow b+\gamma^{a} \zeta^{a}, \\
\zeta^{a} & \rightarrow \zeta^{a}+\hat{\gamma}^{a}, \quad b \rightarrow b+\hat{\gamma}^{a} C^{a} .
\end{aligned}
$$

These PQ symmetries are exact in perturbation theory but broken to discrete subgroups non-perturbatively. In addition, there is the standard T-duality which acts on the radius of the $S^{1}$ and sends $r_{s} \rightarrow r_{s}^{-1}$ while keeping $\lambda_{3}$ fixed. Using (17) and (18) this corresponds to

$$
\lambda_{4} \rightarrow r^{-1}, \quad r \rightarrow \lambda_{4}^{-1},
$$

and also leaves (14) invariant.

\section{M-theory compactified on Calabi-Yau fourfolds}

M-theory has been suggested as the strong coupling limit of type IIA string theory [53]. Even though a satisfactory formulation of M-theory has not been established its low energy limit is known to be 11-dimensional supergravity. In this section we perform the 
compactification of this low energy limit on Calabi-Yau fourfolds and obtain an effective Lagrangian in $D=3$.

The starting point is the 11-dimensional supergravity Lagrangian] [54:

$$
\kappa_{11}^{2} \mathcal{L}^{(11)}=\frac{1}{2} R^{(11)}-\frac{1}{4}\left|F_{4}\right|^{2}-\frac{1}{12}\left(-g^{(11)}\right)^{-1 / 2} A_{3} \wedge F_{4} \wedge F_{4}
$$

where $A_{3}$ is a three-form, $F_{4}$ its field strength and $g^{(11)}$ the determinant of the 11dimensional metric (more details of the notation used are given in appendix A). The Lagrangian (22) is the leading order contribution in a derivative expansion $p / M_{P l}$ where $p$ is the characteristic Fourier momentum. The next term in this expansion has been deduced from a one-loop computation in the type IIA theory 55 and then extrapolated to the 11-dimensional theory. It is associated to the sigma-model anomaly of the 6dimensional fivebrane worldvolume [56]. It reads

$$
\begin{aligned}
\delta \mathcal{L}^{(11)} & \sim \kappa_{11}^{-2 / 3}\left(-g^{(11)}\right)^{-1 / 2} A_{3} \wedge X_{8} \\
\text { with } \quad X_{8} & =\frac{1}{(2 \pi)^{4}}\left(-\frac{1}{768}\left(\operatorname{tr} R^{2}\right)^{2}+\frac{1}{192} \operatorname{tr} R^{4}\right) .
\end{aligned}
$$

(In the following we set $\kappa_{11}=1$.)

In order to do the reduction of (22) let us recall a few facts about Calabi-Yau fourfolds $Y_{4}$ [18. They are Ricci-flat Kähler manifolds of complex dimension four with $S U(4)$ holonomy. The massless modes of the three-dimensional theory are determined by the non-trivial harmonic forms on $Y_{4}$ which are the elements of the cohomology groups $H^{p, q}\left(Y_{4}\right)$. The dimensions $h^{p, q}$ of $H^{p, q}$ satisfy

$$
\begin{aligned}
& h^{p, 0}=h^{0, p}=h^{4, p}=h^{p, 4}=0, \quad p=1,2,3, \\
& h^{0,0}=h^{4,4}=h^{4,0}=h^{0,4}=1, \\
& h^{1,1}=h^{3,3}, \quad h^{1,2}=h^{2,1}=h^{3,2}=h^{2,3}, \quad h^{1,3}=h^{3,1}, \\
& h^{2,2}=2\left(22+2 h^{1,1}+2 h^{1,3}-h^{1,2}\right),
\end{aligned}
$$

leaving $h^{1,1}, h^{1,2}, h^{1,3}$ arbitrary. An important further constraint arises from compactification of the term (23). It induces a potential tadpole term for the three-form $A_{3}$ rendering the resulting vacuum inconsistent [8]. The cofficient of the anomaly is set by the Euler number $\chi$ of $Y_{4}$

$$
\int_{Y_{4}} X_{8}=-\frac{\chi}{24}=-\frac{1}{4}\left(8+h^{1,1}+h^{1,3}-h^{1,2}\right) .
$$

Thus it can be avoided by choosing compactification manifolds with $\chi=0$. However, the anomaly can also be cancelled by considering backgrounds with $n$ space-time filling membranes or turning on non-trival $F_{4}$-flux [7, 8, 17]. 9 In this case

$$
\frac{\chi}{24}=n+\frac{1}{8 \pi^{2}} \int_{Y_{4}} F_{4} \wedge F_{4}
$$

\footnotetext{
${ }^{7}$ The corresponding action is given by $S^{(11)}=\int d^{11} x \sqrt{-g^{(11)}} \mathcal{L}^{(11)}$. Throughout the paper we define the Lagrangian without the factor $\sqrt{-g^{(D)}}$.

${ }^{8} \mathrm{~A}$ further contribution to the anomaly related to M5-branes wrapped around three-cycles in the Calabi-Yau has been discussed in 13 .
} 
has to hold for consistency. Backgrounds with space-time filling membranes are known to be dual to heterotic vacua in non-trivial 5-brane backgrounds [20,26, 41]. Since we are primarily interested in identifying the perturbative heterotic string among the fourfold compactifications we choose to consider $n=0$ in this paper. Furthermore, nontrival $F_{4^{-}}$ flux corresponds in the heterotic dual to discrete twists of the gauge bundle [20,30,40] and can lead to nontrivial torsion [39, 57]. We will analyze this case in a future publication which leaves us with fourfolds that satisfy $\chi=0$ as the compactification manifolds to consider in the following.

We now perform a lowest order Kaluza-Klein reduction of the 11-dimensional supergravity on Calabi-Yau fourfolds.] For the 11-dimensional metric we take the Ansatz

$$
g_{M N}^{(11)}(x, y)=\left(\begin{array}{cc}
g_{\mu \nu}^{(3)}(x) & 0 \\
0 & g_{a b}^{(8)}(x, y)
\end{array}\right),
$$

where $x^{\mu}(\mu=0,1,2)$ denote the coordinates of three-dimensional Minkowski space and $y^{a}(a=3, \ldots, 10)$ denote the internal Calabi-Yau coordinates. In addition $g_{a b}^{(8)}$ depends on a set of moduli $M$ which parameterize the possible deformations of $g_{a b}^{(8)}$ compatible with the Calabi-Yau condition. These moduli appear as massless scalar fields in the threedimensional effective action parameterizing flat directions of the effective potential. In the compactification procedure one chooses an arbitrary point in this moduli space (this corresponds to choosing an arbitrary set of vacuum expectation values for the scalar fields) and expands in the infinitesimal neighborhood of this point $M=\langle M\rangle+\delta M(x)$. This induces

$$
g_{a b}^{(8)}=\hat{g}_{a b}^{(8)}(\langle M\rangle)+\delta g_{a b}^{(8)}(\langle M\rangle, \delta M(x)),
$$

where $\hat{g}_{a b}^{(8)}$ is a background metric and $\delta g_{a b}^{(8)}$ its deformation. Demanding that $\delta g_{a b}^{(8)}$ preserves the Calabi-Yau condition one can expand it in terms of non-trivial harmonic forms on $Y_{4}$. It is convenient to introduce complex coordinates $\xi_{j}(j=1, \ldots, 4)$ for $Y_{4}$ defining $\square \xi_{j}=\frac{1}{\sqrt{2}}\left(y_{2 j-1}+i y_{2 j}\right)$. For the deformation of the Kähler form one has $\square$

$$
i \delta g_{i \bar{\jmath}}=\sum_{A=1}^{h^{1,1}} \delta M^{A}(x) e_{i \bar{\jmath}}^{A},
$$

where $e^{A}$ is an appropriate basis of $H^{1,1}\left(Y_{4}\right)$ and $M^{A}(x)$ are the corresponding (real) moduli. For the deformations of the complex structure one has

$$
\delta g_{\bar{\imath} \bar{\jmath}}=\sum_{\alpha=1}^{h^{3,1}} \delta Z^{\alpha}(x) b_{\bar{\imath} \bar{\jmath}}^{\alpha},
$$

where $Z^{\alpha}(x)$ are complex moduli and $b_{\bar{\imath} \bar{j}}^{\alpha}$ is related to the basis $\Phi^{\alpha}$ of $H^{3,1}\left(Y_{4}\right)$ by an appropriate contraction with the anti-holomorphic 4-form $\bar{\Omega}$ on $Y_{4} 49$ :

$$
b_{\bar{\imath} \bar{\jmath}}^{\alpha}=-\frac{1}{3|\Omega|^{2}} \bar{\Omega}_{\bar{\imath}}^{k l m} \Phi_{k l m \bar{\jmath}}^{\alpha}, \quad|\Omega|^{2} \equiv \frac{1}{4 !} \Omega_{i j k l} \bar{\Omega}^{i j k l} .
$$

\footnotetext{
${ }^{9} \mathrm{~A}$ similar analysis for IIA compactifications on threefolds can be found in ref. [58] while compactification of 11-dimensional supergravity on threefolds was considered in ref. [59, 60].

${ }^{10}$ The corresponding integration measures are related according to $d^{8} \xi \equiv d^{4} \bar{\xi} \wedge d^{4} \bar{\xi}=d^{8} y$.

${ }^{11}$ In the following we omit the superscript (8) at the internal metric.
} 
Finally, the three-form $A_{3}$ is expanded in terms of the (1,1)-forms $e^{A}$ and the (complex) $(2,1)$-forms $\Psi_{i j \bar{k}}^{I}$. More precisely

$$
A_{\mu i \bar{\jmath}}=\sum_{A=1}^{h^{1,1}} A_{\mu}^{A}(x) e_{i \bar{\jmath}}^{A}, \quad A_{i j \bar{k}}=\sum_{I=1}^{h^{2,1}} N^{I}(x) \Psi_{i j \bar{k}}^{I}, \quad A_{\bar{\imath} \bar{\jmath} k}=\sum_{I=1}^{h^{2,1}} \bar{N}^{\bar{J}}(x) \bar{\Psi}_{\bar{\imath} \bar{\jmath} k}^{\overline{\bar{J}}} .
$$

So altogether the compactification leads to $h^{1,1}$ vector multiplets $\left(A_{\mu}^{A}, M^{A}\right)$ and $h^{2,1}+h^{3,1}$ chiral multiplets $\left(N^{I}\right),\left(Z^{\alpha}\right)$.

On the space of $(1,1)$-forms one defines the metric [61]

$$
G_{A B} \equiv \frac{1}{2 \mathcal{V}} \int_{Y_{4}} e^{A} \wedge \star e^{B}=-\frac{1}{2 \mathcal{V}} \int_{Y_{4}} d^{8} \xi \sqrt{g} e_{i \bar{\jmath}}^{A} e_{k \bar{m}}^{B} g^{i \bar{m}} g^{k \bar{\jmath}}
$$

where $\mathcal{V}$ is the volume of $Y_{4}$

$$
\mathcal{V}=\int_{Y_{4}} d^{8} \xi \sqrt{g}=\frac{1}{4 !} \int_{Y_{4}} J \wedge J \wedge J \wedge J=\frac{1}{4 !} d_{A B C D} M^{A} M^{B} M^{C} M^{D} .
$$

$J$ is the Kähler form of the Calabi-Yau fourfold

$$
J=i g_{i \bar{\jmath}} d \xi^{i} \wedge d \bar{\xi}^{\bar{\jmath}}=M^{A} e^{A}
$$

and $d_{A B C D}=\int_{Y_{4}} e^{A} \wedge e^{B} \wedge e^{C} \wedge e^{D}$ are the classical intersection numbers of $Y_{4}$.

On the space of $(3,1)$-forms one defines the metric [50]

$$
G_{\alpha \bar{\beta}} \equiv \frac{\int_{Y_{4}} \Phi^{\alpha} \wedge \bar{\Phi}^{\bar{\beta}}}{\int_{Y_{4}} \Omega \wedge \bar{\Omega}}=\frac{1}{4 \mathcal{V}} \int_{Y_{4}} d^{8} \xi \sqrt{g} b_{\bar{\jmath} \bar{m}}^{\alpha} \bar{b}_{i k}^{\bar{\beta}} g^{i \bar{\jmath}} g^{k \bar{m}}=\partial_{\alpha} \bar{\partial}_{\bar{\beta}} K_{3,1},
$$

which is a Kähler metric with Kähler potential

$$
K_{3,1}=-\ln \left[\int_{Y_{4}} \Omega \wedge \bar{\Omega}\right]
$$

Finally, on the space of $(2,1)$-forms we define a metric $G_{I \bar{J}}$ and intersection numbers $d_{A I \bar{J}}$

$$
\begin{aligned}
G_{I \bar{J}} & \equiv \frac{1}{4} \int_{Y_{4}} \Psi^{I} \wedge \star \Psi^{J}=\frac{1}{8} \int_{Y_{4}} d^{8} \xi \sqrt{g} \Psi_{i j \bar{k}}^{I} \bar{\Psi}_{l \bar{m} \bar{n}}^{\bar{J}} g^{i \bar{m}} g^{j \bar{n}} g^{l \bar{k}} \\
d_{A I \bar{J}} & \equiv \int_{Y_{4}} e^{A} \wedge \Psi^{I} \wedge \bar{\Psi}^{\bar{J}}=\frac{1}{4} \int_{Y_{4}} d^{8} \xi \sqrt{g} \epsilon^{i k l s} \epsilon^{\bar{j} \bar{m} \bar{n} \bar{r}} e_{i \bar{\jmath}}^{A} \Psi_{k l \bar{m}}^{I} \bar{\Psi}_{s \bar{n} \bar{r}}^{\bar{J}}
\end{aligned}
$$

The two quantities are related via

$$
G_{I \bar{J}}=-\frac{i}{4} d_{A I \bar{J}} M^{A}, \quad \text { or } \quad d_{A I \bar{J}}=4 i \frac{\partial G_{I \bar{J}}}{\partial M^{A}} .
$$

${ }^{12}$ This can be checked noticing that $\star \Psi^{J}=\frac{1}{2}\left(\bar{\Psi}_{i \bar{j} \bar{k}}^{\bar{J}} g^{i \bar{\jmath}} g_{l \bar{m}} g_{n \bar{o}}+\bar{\Psi}_{l \bar{k} \bar{m}}^{\bar{J}} g_{n \bar{o}}\right) d \xi^{l} \wedge d \xi^{n} \wedge d \bar{\xi}^{\bar{k}} \wedge d \bar{\xi}^{\bar{m}} \wedge d \bar{\xi}^{\bar{o}}$. The first term corresponds to a term $\tilde{\Psi}^{J} \wedge J \wedge J$ with a globally defined (0,1)-form $\tilde{\Psi}^{J}=\frac{1}{2} \bar{\Psi}_{i \bar{\jmath} \bar{k}}^{\bar{J}} g^{i \bar{\jmath}} d \bar{\xi}^{\bar{k}}$, which is closed because of the harmonicity of $\bar{\Psi}^{\bar{J}}$ and the fact that the Kähler form is covariantly constant with respect to the Hermitian metric. As $h^{0,1}=0$ for a Calabi-Yau fourfold the first term is actually absent. 
In the following it is convenient to define a metric independent of $M^{A}$ as

$$
\hat{G}_{I \bar{J}}=-\frac{i}{4} c^{A} d_{A I \bar{J}}
$$

where $c^{A}$ are constant real vectors with no vanishing entries but otherwise arbitrary.

It is important to notice is that $G_{A B}$ and $d_{A B C D}$ are independent of the complex structure but $G_{I \bar{J}}$ and $d_{A I \bar{J}}$ do depend on $Z^{\alpha}$ and $\bar{Z}^{\bar{\alpha}}$. This dependence is not known generically since it depends on the particular $Y_{4}$ under consideration. However, as we will show it is not necessary to know the complex structure dependence of $G_{I \bar{J}}$ and $d_{A I \bar{J}}$ explicitly in order to determine the Kähler potential.[3]

The basis $\Psi^{I}$ of $(2,1)$-forms can locally be chosen to depend holomorphically on the complex structure or in other words

$$
\bar{\partial}_{\bar{Z}^{\bar{\alpha}}} \Psi^{I}=0, \quad \partial_{Z^{\alpha}} \Psi^{I} \neq 0
$$

Since $h^{3,0}=h^{0,3}=0$ the derivative $\partial_{Z^{\alpha}} \Psi^{I}$ can be expanded into $(1,2)$ - and $(2,1)$-forms with complex-structure dependent coefficient functions $\sigma$ and $\tau$

$$
\partial_{Z^{\alpha}} \Psi^{I}=\sigma_{\alpha I K}(Z, \bar{Z}) \Psi^{K}+\tau_{\alpha I \bar{L}}(Z, \bar{Z}) \bar{\Psi}^{\bar{L}}
$$

Note that $\tau$ is not the complex conjugate of $\sigma$ but an independent function. Differentiating (42) with respect to $\bar{Z}^{\bar{\alpha}}$ results in the following differential constraints for $\sigma$ and $\tau$

$$
\bar{\partial}_{\bar{Z}^{\bar{\beta}}} \sigma_{\alpha I K}=-\tau_{\alpha I \bar{L}} \bar{\tau}_{\bar{\beta} \bar{L} K}, \quad \bar{\partial}_{\bar{Z}^{\bar{\beta}}} \tau_{\alpha I \bar{K}}=-\tau_{\alpha I \bar{L}} \bar{\sigma}_{\bar{\beta} \bar{L} \bar{K}} .
$$

From (38), (39), (40) and (42) it immediatly follows that the complex structure dependence of $G_{I \bar{J}}, G_{I \bar{J}}, d_{A I \bar{J}}$ is constrained by the differential equations

$$
\partial_{Z^{\alpha}} G_{I \bar{J}}=\sigma_{\alpha I K} G_{K \bar{J}}, \quad \partial_{Z^{\alpha}} \hat{G}_{I \bar{J}}=\sigma_{\alpha I K} \hat{G}_{K \bar{J}}, \quad \partial_{Z^{\alpha}} d_{A I \bar{J}}=\sigma_{\alpha I K} d_{A K \bar{J}}
$$

The next step is to insert eqs. (27)-(32) into (22). The details of this reduction are presented in appendix $\mathrm{Q}$ and here we only summarize the results. The vectors $A_{\mu}^{A}$ are again dualized to scalar fields denoted by $P^{A}$. So after dualization the vector multiplet becomes a chiral multiplet with the (real) scalars $\left(M^{A}, P^{A}\right)$ and there are altogether $h^{1,1}+h^{1,2}+h^{1,3}$ chiral multiplets. Supersymmetry requires that the Lagrangian of these chiral multiplets must be expressable in the form

$$
\mathcal{L}^{(3)}=\frac{1}{2} R^{(3)}-G_{\bar{\Lambda} \Sigma} \partial_{\mu} \bar{Z}^{\bar{\Lambda}} \partial^{\mu} Z^{\Sigma}
$$

where $\Lambda, \Sigma=1, \ldots, h^{1,1}+h^{1,2}+h^{1,3}$ and $G_{\bar{\Lambda} \Sigma}$ is a Kähler metric $G_{\bar{\Lambda} \Sigma}=\bar{\partial}_{\bar{\Lambda}} \partial_{\Sigma} K_{\mathrm{M}}^{(3)}$. However, it turns out that the scalar fields which appear naturally in eqs. (29)-(32) in the expansion of the harmonic forms on $Y_{4}$ are not the appropriate Kähler coordinates $Z^{\Sigma}$. Rather a set of field redefinitions has to be performed in order to cast the

\footnotetext{
${ }^{13}$ Similarly, $K_{3,1}$ is generically known only in terms of an integral over the holomorphic $(4,0)$-form $\Omega$ but not necessarily explicitly (see (37)).

${ }^{14}$ It would be nice to have a better geometrical understanding of eqs. (43) and (44).
} 
three-dimensional Lagrangian into the form (45). The proper Kähler coordinates are $T^{A}, \hat{N}^{I}, Z^{\alpha}$ defined as

$$
\begin{aligned}
T^{A} & =\frac{1}{\sqrt{8}}\left(i P^{A}+\mathcal{V} G_{A B} M^{B}-\frac{i}{8} d_{A M \bar{L}} \hat{G}_{\bar{J} M}^{-1} \hat{G}_{\bar{L} I}^{-1} \hat{N}^{I} \overline{\hat{N}}^{\bar{J}}+\omega_{A I K} \hat{N}^{I} \hat{N}^{K}\right), \\
\hat{N}^{I} & =\hat{G}_{I \bar{J}}\left(Z^{\alpha}, \bar{Z}^{\bar{\alpha}}\right) \bar{N}^{\bar{J}},
\end{aligned}
$$

while the $Z^{\alpha}$ are unchanged. The $\omega_{A I K}$ are functions of $Z^{\alpha}$ and $\bar{Z}^{\bar{\alpha}}$ which have to obey

$$
\bar{\partial}_{\bar{Z} \bar{\alpha}} \omega_{A I K}=-\frac{i}{8} \hat{G}_{\bar{L} I}^{-1} \hat{G}_{\bar{J} K}^{-1} d_{A M \bar{L}} \bar{\tau}_{\bar{\alpha} \bar{J} M}
$$

but are otherwise unconstrained. In terms of $T^{A}, \hat{N}^{I}, Z^{\alpha}$ the metric is Kähler with the Kähler potential

$$
K_{\mathrm{M}}^{(3)}=K_{3,1}-\ln \left[\Xi^{A} \mathcal{V} G_{A B}^{-1} \Xi^{B}\right]
$$

where

$$
\Xi^{A} \equiv\left(T^{A}+\bar{T}^{A}+\frac{i}{4 \sqrt{8}} d_{A M \bar{L}} \hat{G}_{\bar{J} M}^{-1} \hat{G}_{\bar{L} I}^{-1} \hat{N}^{I} \overline{\hat{N}}^{\bar{J}}-\frac{1}{\sqrt{8}} \omega_{A I K} \hat{N}^{I} \hat{N}^{K}-\frac{1}{\sqrt{8}} \bar{\omega}_{A \bar{J} \bar{L}} \overline{\hat{N}}^{\bar{J}} \overline{\hat{N}}^{\bar{L}}\right) .
$$

(Note that $\mathcal{V}$ and $G_{A B}$ have to be expressed in terms of the Kähler coordinates.)

Expressed in geometrical terms the argument of the logarithm is just the cube of the volume of the Calabi-Yau fourfold measured in the M-theory metric, which can be checked by inserting (46) into (50)

$$
K_{\mathrm{M}}^{(3)}=K_{3,1}-3 \ln \mathcal{V} .
$$

As we will see in the next section the duality to the heterotic vacua is more naturally expressed in terms of rescaled variables $\tilde{M}^{A}$

$$
M^{A} \rightarrow \tilde{M}^{A}=\mathcal{V}^{1 / 2} M^{A}
$$

Using the rescaled Kähler form $\tilde{J}=\tilde{M}^{A} e^{A}$ in (C.31) and (C.32) one finds

$$
\tilde{\mathcal{V}}=\mathcal{V}^{3}, \quad \tilde{G}_{A B}=\mathcal{V}^{-1} G_{A B},
$$

where $\tilde{\mathcal{V}}$ and $\tilde{G}_{A B}$ have the same functional dependence on $\tilde{M}^{A}$ as $\mathcal{V}$ and $G_{A B}$ have on $M^{A}$. In terms of the rescaled variables the Kähler potential becomes

$$
K_{M}^{(3)}=K_{3,1}-\ln \left[\Xi^{A} \tilde{G}_{A B}^{-1} \Xi^{B}\right]=K_{3,1}-\ln \tilde{\mathcal{V}}
$$

Even though $K_{M}^{(3)}$ is the sum of two terms the moduli space does not factorize. When expressed in terms of the proper Kähler coordinates the second term in eq. (54) does depend on $T^{A}, \hat{N}^{I}, Z^{\alpha}$ and therefore the metric is not block diagonal. However, from eq. (50) we learn that for $\hat{N}^{I}=0$ the moduli space does factorize locally and the Kähler potential becomes

$$
\begin{aligned}
K_{M}^{(3)} & =K_{3,1}+K_{1,1} \\
K_{1,1} & =-\ln \left[\left(T^{A}+\bar{T}^{A}\right) \tilde{G}_{A B}^{-1}\left(T^{B}+\bar{T}^{B}\right)\right] \\
K_{3,1} & =-\ln \left[\int_{Y_{4}} \Omega \wedge \bar{\Omega}\right] .
\end{aligned}
$$


Finally let us discuss the continuous PQ-symmetries of the M-theory vacua in the large volume limit. First of all the scalars $P^{A}$ which arise from dualizing the vectors $A_{\mu}^{A}$ inherit a PQ-symmetry from gauge invariance. The Kähler potential (49) is invariant under the shifts

$$
P^{A} \rightarrow P^{A}+\tilde{\gamma}^{A}
$$

where $\tilde{\gamma}^{A}$ are arbitrary real constants. Secondly, the $N^{I}$ arise from expanding the threeform in eq. (32) and as a consequence they 'inherit' part of the three-form gauge invariance. Specifically, the three-dimensional Lagrangian is invariant under the shift

$$
N^{I} \Psi^{I}+\bar{N}^{\bar{I}} \bar{\Psi}^{\bar{I}} \rightarrow N^{I} \Psi^{I}+\bar{N}^{\bar{I}} \bar{\Psi}^{\bar{I}}+\text { const. . }
$$

In terms of $N^{I}$ this amounts to

$$
N^{I} \rightarrow N^{I}+\gamma^{I}(Z, \bar{Z}), \quad \bar{N}^{\bar{I}} \rightarrow \bar{N}^{\bar{I}}+\bar{\gamma}^{\bar{I}}(Z, \bar{Z}),
$$

where the $\gamma^{I}$ depend on the complex structure and as a consequence of (42) have to satisfy

$$
\partial_{Z^{\alpha}} \gamma^{J}=-\gamma^{I} \sigma_{\alpha I J}, \quad \bar{\partial}_{\bar{Z}_{\bar{\alpha}}} \gamma^{J}=-\bar{\gamma}^{\bar{I}} \bar{\tau}_{\bar{\alpha} \bar{I} J} .
$$

The redefinition of the $N^{I}$ in eq. (47) is precisely such that it renders the PQ-symmetry holomorphic

$$
\hat{N}^{I} \rightarrow \hat{N}^{I}+\hat{\gamma}^{I}(Z)
$$

where $\hat{\gamma}^{I}=\hat{G}_{I \bar{J}} \bar{\gamma}^{\bar{J}}$ obeys

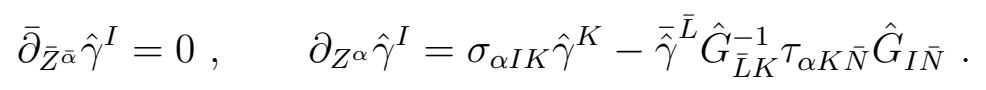

The corresponding PQ transformations of the fields $T^{A}$ are

$$
\begin{aligned}
T^{A} \rightarrow & T^{A}-\frac{i}{4 \sqrt{8}} d_{A M \bar{L}} \hat{G}_{\bar{J} M}^{-1} \hat{G}_{\bar{L} I}^{-1} \hat{N}^{I} \bar{\gamma}^{\bar{J}}-\frac{i}{8 \sqrt{8}} d_{A M \bar{L}} \hat{G}_{\bar{J} M}^{-1} \hat{G}_{\bar{L} I}^{-1} \hat{\gamma}^{I} \overline{\hat{\gamma}}^{\bar{J}} \\
& +\frac{1}{\sqrt{8}} \omega_{A I K} \hat{\gamma}^{I} \hat{N}^{K}+\frac{1}{2 \sqrt{8}} \omega_{A I K} \hat{\gamma}^{I} \hat{\gamma}^{K}+\frac{1}{\sqrt{8}} \bar{\omega}_{A \bar{I} \bar{K}} \overline{\hat{\gamma}}^{\bar{I}} \overline{\hat{N}}^{\bar{K}}+\frac{1}{2 \sqrt{8}} \bar{\omega}_{A \bar{I} \bar{K}} \overline{\hat{\gamma}}^{\bar{I}} \overline{\hat{\gamma}}^{\bar{K}} .
\end{aligned}
$$

Thus the $P^{A}$ also transform四 according to

$$
P^{A} \rightarrow P^{A}-\frac{1}{8} d_{A M \bar{L}} \hat{G}_{\bar{J} M}^{-1} \hat{G}_{\bar{L} I}^{-1} \hat{N}^{I} \overline{\hat{\gamma}}^{\bar{J}}+\frac{1}{8} d_{A M \bar{L}} \hat{G}_{\bar{J} M}^{-1} \hat{G}_{\bar{L} I}^{-1} \hat{\gamma}^{I} \overline{\hat{N}}^{\bar{J}} .
$$

So altogether we have $h^{1,1}+2 h^{2,1}$ continuous PQ-symmetries in the large volume limit of the M-theory compactification.

\section{Duality}

\subsection{Heterotic - M-theory duality in $D=3$}

The next step is to investigate the dual relation between the heterotic and the M-theory vacua. A first guidance can be obtained by considering the 7 -dimensional duality (M/K3

\footnotetext{
${ }^{15}$ This transformation is necessary in order to render (C.38) invariant under (60).
} 
$\simeq$ Het $/ \mathrm{T}^{3}$ ] [53] and fibering it over a common complex 2-dimensional base $B_{2}$. For large $B_{2}$ one can apply the adiabatic argument 62] and conclude that M-theory compactified on a K3-fibred fourfold should be dual to the heterotic string compactified on an elliptically fibred threefold. The 7-dimensional string coupling constant $\lambda_{7}$ is related to the volume $\mathcal{V}_{K 3}$ of the $\mathrm{K} 3$ measured in the (11-dimensional) M-theory metric and the respective space-time metrics $\left(g_{7}^{\text {het }}, g_{7}^{M}\right)$ differ by a power of $\lambda_{7}$ [53]

$$
\lambda_{7}^{4 / 3}=\mathcal{V}_{K 3}, \quad g_{7}^{\text {het }}=\lambda_{7}^{4 / 3} g_{7}^{M} .
$$

Using the adiabatic argument one derives

$$
\frac{1}{\lambda_{3}^{2}}=\frac{\mathcal{V}_{B_{2}}^{\text {het }}}{\lambda_{7}^{2}}=\lambda_{7}^{2 / 3} \mathcal{V}_{B_{2}}=\mathcal{V}_{K 3}^{1 / 2} \mathcal{V}_{B_{2}}
$$

where the volume of the base $B_{2}$ measured in the heterotic string frame metric $\mathcal{V}_{B_{2}}^{\text {het }}$ is related to the volume $\mathcal{V}_{B_{2}}$ measured in the M-theory metric via $\mathcal{V}_{B_{2}}^{\text {het }}=\lambda_{7}^{8 / 3} \mathcal{V}_{B_{2}}$ as can bee seen from eq. (64). Eq. (65) implies that a large volume of the Calabi-Yau fourfold corresponds to heterotic weak coupling. Since the compactification of M-theory performed in the previous section is valid for large fourfolds it is legitimate to compare it to a weakly coupled heterotic string. Or in other words the two limits - large $Y_{4}$ in Mtheory and weak coupling in the heterotic string - are mutually compatible. In particular duality requires that the respective Kähler potentials have to agree in this limit

$$
K_{\mathrm{M}}^{(3)}=K_{\mathrm{het}}^{(3)} .
$$

For perturbative heterotic vacua where $K_{\text {het }}^{(3)}$ is given by eq. (12) the equality between the Kähler potentials is not automatically satisfied but rather puts a constraint on the intersection numbers $d_{A B C D}$ or more generally on the Calabi-Yau fourfold. It would be desirable to find those fourfolds which correspond to a perturbative heterotic vacuum exactly as was done in ref. [45] for type IIA compactified on Calabi-Yau threefolds. In this paper we only make the first step in this direction in that we show that for the $(1,1)$ moduli of K3-fibred fourfolds eq. (66) holds in the large base limit.

As the base $B_{2}$ we take in both theories a Hirzebruch surface $\mathbb{F}_{n}$ with $n$ even and freeze the values of the scalars $\hat{N}^{I}, Z^{\alpha}$ on the M-theory side. In this case the M-theory Kähler potential simplifies $K_{\mathrm{M}}^{(3)}=K_{1,1}$, where $K_{1,1}$ is given in eq. (55). The particular geometry of the Calabi-Yau fourfold we are considering affects the form of its intersection numbers and thus in view of (C.32) also the form of $\tilde{G}_{A B}$. But (C.32) determines $\tilde{G}_{A B}$ in terms of the coordinates $\tilde{M}^{A}$. In order to find a map between the heterotic and the M-theory variables one has to compare the Kähler potentials expressed in the Kähler coordinates. In general it is not possible to invert (46) and explicitly express $\tilde{M}^{A}$ (and thus $\tilde{G}_{A B}$ ) in terms of the $T^{A}$. However, as we will see shortly the explicit relation between $\tilde{M}^{A}$ and $T^{A}$ can be obtained in the large base limit.

In this limit the intersection numbers of $Y_{4}$ enjoy specific properties. We therefore need to know the group of its divisors ${ }^{\mathbb{1}}$, i.e. $H_{6}\left(Y_{4}\right)$. Let $\pi: Y_{4} \rightarrow B_{2}$ denote the projection map of the K3 fibred fourfold. One has three different contributions to $H_{6}\left(Y_{4}\right)$ :

\footnotetext{
${ }^{16} \mathrm{~A}$ similar analysis for K3 fibred threefolds can be found in section 5.5 of [63].
} 
1. Each divisor $C$ of the base $B_{2}$ contributes a divisor $D$ of $Y_{4}$ via its pullback $D=\pi^{\star} C$.

2. Each monodromy invariant algebraic 2-cycle $G_{i}$ in the generic fibre $F_{0}$ gives a divisor $D_{i}$ of $Y_{4}$ if it is transported around the base.

3. When the K3 fibre degenerates over a locus of codimension one in the base in such a way that the degenerate fibre is reducible, the volumes of its components can be varied independently. Therefore such reducible "bad fibres" $B_{a}$ contribute further elements to $H_{6}\left(Y_{4}\right)$.

The intersection of two divisors of the first kind can be traced back to the intersection of the corresponding divisors in the base:

$$
\left(D_{1} \cdot D_{2}\right)_{Y_{4}}=\left(\pi^{\star} C_{1} \cdot \pi^{\star} C_{2}\right)_{Y_{4}}=\pi^{\star}\left(C_{1} \cdot C_{2}\right)_{B_{2}},
$$

which is not a number, but a four-cycle in the Calabi-Yau fourfold. The subscript indicates the space within which we are considering the intersection theory. As explained in $4 \mathbb{F}_{n}$ (with $n$ even) is topologically the product of two $S^{2}$ 's, whose areas are the two Kähler parameters $U$ and $V$. The corresponding divisors $C_{U}$ and $C_{V}$ have the intersection numbers $\left(C_{U} \cdot C_{U}\right)_{B_{2}}=\left(C_{V} \cdot C_{V}\right)_{B_{2}}=0$ and $\left(C_{U} \cdot C_{V}\right)_{B_{2}}=1$. In view of (67) the intersections of the two related divisors of the fourfold are either zero or the generic fibre, i.e. $\left(D_{U} \cdot D_{U}\right)_{Y_{4}}=0,\left(D_{V} \cdot D_{V}\right)_{Y_{4}}=0$ and $\left(D_{U} \cdot D_{V}\right)_{Y_{4}}=F_{0}$. From this fact follows that the intersection of three and four $D_{U}, D_{V}$ automatically vanishes and that $D_{U} \cdot D_{V}$ has no intersection with divisors coming from bad fibres $B_{a}$. Finally, we have

$$
\left(D_{U} \cdot D_{V} \cdot D_{i} \cdot D_{j}\right)_{Y_{4}}=\left(G_{i} \cdot G_{j}\right)_{F_{0}}
$$

where $G_{i}, G_{j}$ and $D_{i}, D_{j}$ are defined in the second entry of the list above. In particular $G_{i}$ and $G_{j}$ are dual to elements of the (monodromy invariant) part of the Picard lattice of the generic fibre which has signature $(+,-, \ldots,-)$ 63]. Thus it is possible to choose the divisors of $F_{0}$ in a way that the right hand side of (68) is given by $\eta_{i j}=(+,-, \ldots,-)$.

In the large base limit the (rescaled) volume $\tilde{\mathcal{V}}=\frac{1}{4 !} d_{A B C D} \tilde{M}^{A} \tilde{M}^{B} \tilde{M}^{C} \tilde{M}^{D}$ is dominated by those terms which contain a maximal number of base moduli. From what we have just said it is clear that the leading contribution is

$$
\tilde{\mathcal{V}}=\frac{1}{2} d_{U V i j} \tilde{U} \tilde{V} \tilde{M}^{i} \tilde{M}^{j}=\frac{1}{2} \tilde{U} \tilde{V} \eta_{i j} \tilde{M}^{i} \tilde{M}^{j}
$$

where $\tilde{U} \tilde{V}$ is the (rescaled) volume of the base and $\frac{1}{2} \eta_{i j} \tilde{M}^{i} \tilde{M}^{j}$ that of the generic fibre. Obviously we are in an adiabatic regime, in which to leading order the volume is given by the product of the base and fibre volumes $\tilde{\mathcal{V}}_{Y_{4}}=\tilde{\mathcal{V}}_{B_{2}} \tilde{\mathcal{V}}_{\tilde{\sigma} 3}$. Using the intersection numbers (69) and (C.32) we can compute $\tilde{G}_{A B}$ for the moduli $\tilde{U}$ and $\tilde{V}$ of the base and the moduli $M^{i}$ of the generic fibre in the large base limit'

$$
\tilde{G}_{A B}=\left(\begin{array}{ccc}
\frac{1}{2 \tilde{U}^{2}} & 0 & 0 \\
0 & \frac{1}{2 \tilde{V}^{2}} & 0 \\
0 & 0 & -\frac{\eta_{i j}}{\eta_{k l} \tilde{M}^{k} \tilde{M}^{l}}+\frac{2 \eta_{i k} \tilde{M}^{k} \eta_{j l} \tilde{M}^{l}}{\left(\eta_{k l} \tilde{M}^{k} \tilde{M}^{l}\right)^{2}}
\end{array}\right)
$$

\footnotetext{
${ }^{17}$ At this point we neglect the possibility of moduli arising from bad fibres and discuss their contribution at the end of the section.
} 
Inserted into (46) using (53) one obtains

$$
\begin{aligned}
T^{U}+\bar{T}^{U} & =\frac{1}{4}\left(\frac{\tilde{V}}{\tilde{U}}\right)^{1 / 2}\left(\eta_{k l} \tilde{M}^{k} \tilde{M}^{l}\right)^{1 / 2} \\
T^{V}+\bar{T}^{V} & =\frac{1}{4}\left(\frac{\tilde{U}}{\tilde{V}}\right)^{1 / 2}\left(\eta_{k l} \tilde{M}^{k} \tilde{M}^{l}\right)^{1 / 2} \\
T^{i}+\bar{T}^{i} & =\frac{1}{2}(\tilde{U} \tilde{V})^{1 / 2} \frac{\eta_{i j} \tilde{M}^{j}}{\left(\eta_{k l} \tilde{M}^{k} \tilde{M}^{l}\right)^{1 / 2}} .
\end{aligned}
$$

In connection with (69) this implies

$$
\tilde{\mathcal{V}}=32\left(T^{U}+\bar{T}^{U}\right)\left(T^{V}+\bar{T}^{V}\right)\left(T^{i}+\bar{T}^{i}\right) \eta_{i j}\left(T^{j}+\bar{T}^{j}\right)
$$

Thus in the large $B_{2}$ limit the volume also factorizes in the Kähler coordinates $T^{A}$. The M-theory Kähler potential reads in this limit

$$
K_{\mathrm{M}}^{(3)}=-\ln \tilde{\mathcal{V}}=-\ln \left[\left(T^{U}+\bar{T}^{U}\right)\left(T^{V}+\bar{T}^{V}\right)\left(T^{i}+\bar{T}^{i}\right) \eta_{i j}\left(T^{j}+\bar{T}^{j}\right)\right],
$$

and can now be compared to the $K_{\text {het }}^{(3)}$ of the heterotic vacua specified in eq. (15).

On the heterotic side we take the large base limit together with the large $S$-limit (weak four-dimensional coupling). Thus only $S^{\prime}, T^{\prime}, D^{a}$ and the $(1,1)$ moduli $U, V$ of the base $B_{2}$ have to be taken into account while all other $(1,1)$ moduli, all $(2,1)$ and all gauge bundle moduli can be frozen at generic values. In elliptically fibred threefolds $Y_{3}$ with the Hirzebruch surface $\mathbb{F}_{n}$ as a base the Kähler potential of $U$ and $V$ simplifies to $\tilde{K}^{(4)}=-\ln [(U+\bar{U})(V+\bar{V})][$, 64]. So altogether we have on the heterotic side

$$
K_{\text {het }}^{(3)}=-\ln [(U+\bar{U})(V+\bar{V})]-\ln \left[\left(S^{\prime}+\bar{S}^{\prime}\right)^{2}-\left(T^{\prime}+\bar{T}^{\prime}\right)^{2}-\left(D^{a}+\bar{D}^{a}\right)^{2}\right] .
$$

The two Kähler potentials agree if one identifies

$$
\left(S^{\prime}, T^{\prime}, D^{a}\right) \leftrightarrow\left(T^{i}\right), \quad(U, V) \leftrightarrow\left(T^{U}, T^{V}\right)
$$

We see that the moduli which parameterize the base $B_{2}$ are identified on both sides and the $(1,1)$ moduli of the generic K3-fibre on the M-theory side are identified with the four-dimensional dilaton, the radius of $S^{1}$ and the scalars $D^{a}$ related to the heterotic vector multiplets. Note that the correspondence (75) implies according to eq. (19) and (71)

$$
\lambda_{3}^{-4} \sim\left(T^{i}+\bar{T}^{i}\right) \eta_{i j}\left(T^{j}+\bar{T}^{j}\right) \sim \tilde{\mathcal{V}}_{B_{2}}=\mathcal{V}_{B_{2}} \mathcal{V}
$$

which agrees with (65) obtained in the adiabatic regime. Furthermore the number of moduli $T^{i}$ on the M-theory side is bounded by the maximal rank of the Picard group of the generic fibre, i.e. by $h^{(1,1)}\left(F_{0}\right)=20$. This is consistent with the bound on the heterotic gauge group given in (3). In fact it is a little lower but that could be related to the fact that our analysis is based on purely classical geometry. (The same issue arises in type IIA vacua compactified on threefolds 45.)

So far we neglected moduli arising from reducible bad K3 fibres in the fourfold, i.e. we considered no divisors of the third kind. In the context of the duality between the 
heterotic string compactified on $K 3 \times T^{2}$ and the type IIA string on K3-fibred $Y_{3}$ the reducible bad K3 fibres were shown to be related to non-perturbative physics on the heterotic side [45]. The same is true for M- and F-theory compactifications on K3-fibred threefolds $Y_{3}$ [4, 5]. For those K3-fibred fourfolds $Y_{4}$ which can be adiabatically obtained as K3-fibred threefolds over $\mathbb{P}^{1}$ it follows that the reducible bad K3 fibres also correspond to non-perturbative physics on the heterotic side [33. This can be explicitly checked in the Kähler potentials 65. Finally, the identification of the $(2,1)$ and $(3,1)$ moduli of $Y_{4}$ with the elliptic fibre, the $(2,1)$ and the bundle moduli of $Y_{3}$ on the heterotic side is discussed in refs. 20, 21, 25, 30, 33.

\section{$4.2 \quad$ F-theory limit}

Ultimately, one is interested in lifting the M-theory/heterotic duality discussed so far to four space-time dimensions. This amounts to a simple decompactification on the heterotic side while in the dual theory one has to take the F-theory limit [3]. The following discussion of the F-theory limit is strongly inspired by ref. [63] where a similar decompactification limit is discussed in the context of type IIA/heterotic duality in four dimensions.

Taking the F-theory limit requires that the Calabi-Yau fourfold is elliptically fibred. Here we focus on those fourfolds which are in addition K3-fibred. Thus the F-theory limit for this restricted class of fourfolds requires K3-fibres which themselves are elliptically fibred over a base $\mathbb{P}^{1}$. The first step is to blow down any rational curves in the generic K3-fibre which take the Picard number above its minimal value of 2. (The two moduli which are always present arise from the base $\mathbb{P}^{1}$ and the elliptic fibre.) The map (75) implies that on the heterotic side this corresponds to freezing the scalars corresponding to the real part of $D^{a}$. After blowing down the Picard lattice is given by the even self-dual lattice $\Gamma_{1,1}$, which we supose to be generated by the null vector $v$ and its dual $v^{\star}$. The Kähler form of the generic K3-fibre is given by

$$
J=\sqrt{\frac{1}{2 \beta}} v^{\star}+\sqrt{\frac{\beta}{2}} v, \quad \beta \in \mathbb{R}^{+},
$$

where the coefficients have been chosen such that the volume of the generic fibre in Mtheory units is of order one. As we are only interested in the qualitative features of the F-theory limit we do not keep track of any constants of order one in the following. The class of the base of the K3 is given by $v^{\star}-v$ (having self-intersection number -2 ) and the class of the elliptic fibre by $v$ (with self-intersection number 0 ). The choice $d_{U V i j}=$ $\eta_{i j}=(+,-, \ldots,-)$ in our previous discussion corresponds to choosing the divisors of the generic K3-fibre in such a way that the forms representing the cohomology classes of the Poincaré duals of these divisors are given by $A=\frac{1}{\sqrt{2}}\left(v+v^{\star}\right)$ and $B=\frac{1}{\sqrt{2}}\left(v-v^{\star}\right)$. One verifies that they obey $A . A=1, B \cdot B=-1$ and $A \cdot B=0 . \quad\left(A \cdot A \equiv \int_{K 3} A \wedge A\right.$, etc. $)$ Expanded in this basis the Kähler form of the generic K3-fibre (77) is given by

$$
J=\frac{1}{2}\left(\frac{1}{\sqrt{\beta}}+\sqrt{\beta}\right) A+\frac{1}{2}\left(\sqrt{\beta}-\frac{1}{\sqrt{\beta}}\right) B .
$$

\footnotetext{
${ }^{18}$ Strictly speaking we should first undo the duality transformation which related the three-dimensional vector multiplets to chiral multiplets since in the F-theory limit the four-dimensional vector multiplets (which have no scalar) have to be recovered.
} 
This implies the identification $M^{1}=\frac{1}{2}\left(\frac{1}{\sqrt{\beta}}+\sqrt{\beta}\right)$ and $M^{2}=\frac{1}{2}\left(\sqrt{\beta}-\frac{1}{\sqrt{\beta}}\right)$. Inserted into eq. (71) using (76) and the fact that the volume of the K3 is of order one we derive

$$
\begin{aligned}
T^{1}+\bar{T}^{1} & \sim \lambda_{3}^{-2}\left(\sqrt{\beta}+\frac{1}{\sqrt{\beta}}\right) \\
T^{2}+\bar{T}^{2} & \sim-\lambda_{3}^{-2}\left(\sqrt{\beta}-\frac{1}{\sqrt{\beta}}\right) .
\end{aligned}
$$

From the map (75) we expect $S^{\prime}=\frac{1}{2}(S+T) \leftrightarrow T^{1}$ and $T^{\prime}=\frac{1}{2}(S-T) \leftrightarrow T^{2}$. Thus we obtain

$$
\begin{aligned}
\lambda_{4}^{-2} & \sim S+\bar{S} \sim\left(T^{1}+\bar{T}^{1}\right)+\left(T^{2}+\bar{T}^{2}\right) \sim \lambda_{3}^{-2} \frac{1}{\sqrt{\beta}} \\
r^{2} & \sim T+\bar{T} \sim\left(T^{1}+\bar{T}^{1}\right)-\left(T^{2}+\bar{T}^{2}\right) \sim \lambda_{3}^{-2} \sqrt{\beta} .
\end{aligned}
$$

The volume of the elliptic fibre is given by

$$
J . v=\frac{1}{\sqrt{2 \beta}},
$$

which has to be taken to zero in the F-theory limit [3]. In view of (82) this is equivalent to $\beta \rightarrow \infty$. On the heterotic side it corresponds to the expected decompactification limit $r \rightarrow \infty$ (with $r \lambda_{3}^{2}=\lambda_{4}^{2}$ fixed) as can be seen from (81). Finally, we learn from (16) and (80) that $\sqrt{\beta} \sim r_{s}$.

\section{Conclusions}

In this paper we derived the Kähler potentials for the three-dimensional perturbative heterotic string (in terms of the four-dimensional one) and the Kähler potential for the moduli of a Calabi-Yau fourfold in the large volume limit of M-theory including the moduli coming from the three-form potential. We showed that both have a common range of validity (weak heterotic coupling and large volume of the fourfold are compatible with each other) and that they do agree for K3-fibred fourfolds and elliptic threefolds in the large base limit.

It would be desirable to generalize this analysis and determine all fourfolds which obey eq. ([6]). Moreover, our analysis was restricted to fourfolds with vanishing Euler number $(\chi=0)$ corresponding to the absence of space-time filling membranes and nontrivial four-form flux. Incorporating such effects requires the presence of a non-trivial warp factor in the Ansatz for the metric (27) [7].

Perturbative quantum corrections on the heterotic side which correct the gauge kinetic function $f_{a b}$ and the Kähler potential can be taken into account and related to 'geometrical corrections' on the M-theory side. In this manner one might derive a geometrical version of the heterotic perturbation expansion [65]. In particular, the holomorphic $f$ function has to be related to holomorphic quantities of the fourfold. Closely related is the issue of non-perturbative corrections to the superpotential which corresponds to wrapping M5-branes over appropriate six-cycles in the fourfold [6]. 
Finally, in $D=2$ the duals of the heterotic vacua are related to IIA compactified on $Y_{4}$. This duality might be used to leave the regime of large $Y_{4}$ and compute stringy $\left(\alpha^{\prime}\right)$ corrections. Similarly, issues of mirror symmetry in Calabi-Yau fourfolds can be studied.

\section{Acknowledgements}

We would like to thank P. Aspinwall, T. Bauer, R. Böhm, G. Curio, H. Günther, B. Hunt, W. Lerche, W. Lucht, D. Lüst, P. Mayr, T. Mohaupt, A. Sen, S. Stieberger and A. Strominger for useful discussions. J.L. thanks the ITP, Santa Barbara for hospitality while part of this work was done.

This work is supported by GIF (the German-Israeli Foundation for Scientific Research), DAAD (the German Academic Exchange Service) and the Landesgraduiertenförderung Sachsen-Anhalt.

\section{Appendix}

\section{A Notation and conventions}

The signature of the space-time metric is $(-+\ldots+)$. The Levi-Civita symbol is defined to transform as a tensor, i.e. we have

$$
\epsilon^{1 \ldots D}=\left( \pm g^{(D)}\right)^{-1 / 2} \quad \text { and } \quad \epsilon_{1 \ldots D}=\left( \pm g^{(D)}\right)^{1 / 2},
$$

where the '-'-sign has to be used for space-time indices while on the internal Calabi-Yau manifold the '+'-sign is appropriate. Our conventions for the Riemann curvature tensor are

$$
R_{\nu \rho \sigma}^{\mu}=\partial_{\rho} \Gamma_{\nu \sigma}^{\mu}-\partial_{\sigma} \Gamma_{\nu \rho}^{\mu}+\Gamma_{\nu \sigma}^{\omega} \Gamma_{\omega \rho}^{\mu}-\Gamma_{\nu \rho}^{\omega} \Gamma_{\omega \sigma}^{\mu},
$$

where we use the following definition of the Christoffel symbols:

$$
\Gamma_{\nu \rho}^{\mu}=\frac{1}{2} g^{\mu \sigma}\left(\partial_{\nu} g_{\sigma \rho}+\partial_{\rho} g_{\sigma \nu}-\partial_{\sigma} g_{\nu \rho}\right) .
$$

The Ricci tensor is defined as

$$
R_{\mu \nu}=R_{\mu \rho \nu}^{\rho} .
$$

(We are thus using the $(+++)$ conventions of 66].)

Furthermore a $p$-form $A_{p}$ can be expanded as

$$
A_{p}=\frac{1}{p !} A_{\mu_{1} \ldots \mu_{p}} d x^{\mu_{1}} \wedge \ldots \wedge d x^{\mu_{p}},
$$

which has an obvious generalisation to $(p, q)$-forms:

$$
A_{p, q}=\frac{1}{p ! q !} A_{i_{1} \ldots i_{p} \overline{1}_{1} \ldots \bar{i}_{q}} d \xi^{i_{1}} \wedge \ldots \wedge d \xi^{i_{p}} \wedge d \bar{\xi}^{\bar{\xi}_{1}} \wedge \ldots \wedge d \overline{\bar{\xi}} .
$$


The exterior derivative is defined as

$$
d A_{p}=\frac{1}{p !} \partial_{\mu} A_{\mu_{1} \ldots \mu_{p}} d x^{\mu} \wedge d x^{\mu_{1}} \wedge \ldots \wedge d x^{\mu_{p}},
$$

which entails because of

$$
F_{p+1}=d A_{p}=\frac{1}{(p+1) !} F_{\mu_{1} \ldots \mu_{p+1}} d x^{\mu_{1}} \wedge \ldots \wedge d x^{\mu_{p+1}}
$$

the relation

$$
F_{\mu_{1} \ldots \mu_{p+1}}=(p+1) \partial_{\left[\mu_{1}\right.} A_{\left.\mu_{2} \ldots \mu_{p+1}\right]} .
$$

With this definition the action for a $p$-form potential $A_{p}$ is given by

$$
-\frac{1}{4} \int d^{D} x \sqrt{-g^{(D)}}\left|F_{p+1}\right|^{2}=-\frac{1}{4} \int d^{D} x \frac{\sqrt{-g^{(D)}}}{(p+1) !} F_{\mu_{1} \ldots \mu_{p+1}} F^{\mu_{1} \ldots \mu_{p+1}} .
$$

The Hodge star operator for a $p$-form is defined as

$$
\star A_{p}=\frac{1}{p !(D-p) !} A_{\mu_{1} \ldots \mu_{p}} \epsilon^{\mu_{1} \ldots \mu_{p}}{ }_{\nu_{p+1} \ldots \nu_{D}} d x^{\nu_{p+1}} \wedge \ldots \wedge d x^{\nu_{D}} .
$$

It is generalised to $(p, q)$-forms on a Hermitian manifold (where $D$ now denotes its complex dimension) by

$$
\begin{array}{r}
\star A_{p, q}=\frac{(-1)^{(D-p) q}}{p ! q !(D-p) !(D-q) !} \bar{A}_{i_{1} \ldots i_{q} \bar{\tau}_{1} \ldots \bar{i}_{p}} \epsilon^{i_{1} \ldots i_{q} \bar{\jmath}_{q+1} \ldots \bar{j}_{D}} \epsilon^{\overline{1}_{1} \ldots \bar{\tau}_{p}}{ }_{j_{p+1} \ldots j_{D}} \\
\times d \xi^{j_{p+1}} \wedge \ldots \wedge d \xi^{j_{D}} \wedge d \bar{\xi}^{\bar{\jmath}_{q+1}} \wedge \ldots \wedge d \bar{\xi}^{\bar{j}_{D}}
\end{array}
$$

where $\bar{A}_{i_{1} \ldots i_{q} \bar{\tau}_{1} \ldots \bar{i}_{p}} \equiv \overline{A_{i_{1} \ldots i_{p} \bar{p}_{1} \ldots \bar{q}_{q}}}$. This definition ensures that we have the following expression for the scalar product of two $(p, q)$-forms on a Hermitian manifold:

$$
\begin{aligned}
\left(A_{p, q}, B_{p, q}\right) & \equiv \frac{1}{p ! q !} \int d^{D} \xi d^{D} \bar{\xi} \sqrt{g^{(D)}} A_{i_{1} \ldots i_{p} \bar{\imath}_{1} \ldots \bar{i}_{q}} \bar{B}^{\bar{\iota}_{1} \ldots \bar{q}_{q} i_{1} \ldots i_{p}} \\
& =\int A_{p, q} \wedge \star B_{p, q} .
\end{aligned}
$$

\section{B $S^{1}$ compactification of $D=4$ supergravity}

In this appendix we give some details of the $S^{1}$-reduction of four-dimensional supergravity which follows rather closely ref. [52]. Inserting (9) into (1) and performing a Weyl rescaling $g_{\mu \nu}^{(3)} \rightarrow r^{2} g_{\mu \nu}^{(3)}$ one arrives at

$$
\begin{aligned}
\mathcal{L}^{(3)} & =\frac{1}{2} R^{(3)}-\frac{1}{r^{2}} \partial_{\mu} r \partial^{\mu} r-G_{\bar{I} J} \partial_{\mu} \bar{\Phi}^{\bar{I}} \partial^{\mu} \Phi^{J}+\frac{r^{4}}{4} H_{\mu} H^{\mu}-\frac{1}{2 r^{2}}(\operatorname{Re} f)_{a b} \partial_{\mu} \zeta^{a} \partial^{\mu} \zeta^{b} \\
& +\frac{r^{2}}{2}(\operatorname{Re} f)_{a b}\left(F_{\mu}^{a}+\zeta^{a} H_{\mu}\right)\left(F^{b \mu}+\zeta^{b} H^{\mu}\right)+(\operatorname{Im} f)_{a b} \partial_{\mu} \zeta^{a}\left(F^{b \mu}+\zeta^{b} H^{\mu}\right),
\end{aligned}
$$


where the following abbreviations are used:

$$
H^{\mu}=\frac{1}{2} \epsilon^{\mu \nu \rho} H_{\nu \rho}=\frac{1}{2} \epsilon^{\mu \nu \rho}\left(\partial_{\nu} B_{\rho}-\partial_{\rho} B_{\nu}\right), \quad F^{a \mu}=\frac{1}{2} \epsilon^{\mu \nu \rho} F_{\nu \rho}^{a}=\frac{1}{2} \epsilon^{\mu \nu \rho}\left(\partial_{\nu} A_{\rho}^{a}-\partial_{\rho} A_{\nu}^{a}\right) .
$$

The vectors can be dualised to scalars by adding $\mathrm{r}+1$ Lagrange multipliers $C^{a}$ and $b$ to the Lagrangian of eq. (B.14)

$$
\mathcal{L}^{(3)} \rightarrow \mathcal{L}^{(3)}-F_{\mu}^{a} \partial^{\mu} C^{a}+\frac{1}{2} H_{\mu} \partial^{\mu}\left(b-\zeta^{a} C^{a}\right),
$$

and eliminate the fields $F_{\mu}^{a}$ and $H_{\mu}$ via their equations of motion. This results in

$$
\begin{aligned}
\mathcal{L}^{(3)} & =\frac{1}{2} R^{(3)}-\frac{1}{r^{2}} \partial_{\mu} r \partial^{\mu} r-G_{\bar{I} J} \partial_{\mu} \bar{\Phi}^{\bar{I}} \partial^{\mu} \Phi^{J}-\frac{1}{2 r^{2}}(\operatorname{Re} f)_{a b} \partial_{\mu} \zeta^{a} \partial^{\mu} \zeta^{b} \\
& -\frac{1}{4 r^{4}}\left(\partial_{\mu} b+\zeta^{a} \stackrel{\leftrightarrow}{\partial}_{\mu} C^{a}\right)^{2}-\frac{1}{2 r^{2}}\left(\partial_{\mu} C^{a}-(\operatorname{Im} f)_{a c} \partial_{\mu} \zeta^{c}\right)(\operatorname{Re} f)_{a b}^{-1}\left(\partial^{\mu} C^{b}-(\operatorname{Im} f)_{b d} \partial^{\mu} \zeta^{d}\right)
\end{aligned}
$$

Expressed in the Kähler coordinates (10) $\mathcal{L}^{(3)}$ takes the form

$$
\begin{aligned}
\mathcal{L}^{(3)} & =\frac{1}{2} R^{(3)}-G_{\bar{I} J} \partial_{\mu} \bar{\Phi}^{\bar{I}} \partial^{\mu} \Phi^{J} \\
& -\frac{\left|\partial_{\mu} T-(D+\bar{D})^{a}(\operatorname{Re} f)_{a b}^{-1} \partial_{\mu} D^{b}+\frac{1}{4}(D+\bar{D})^{a}(\operatorname{Re} f)_{a c}^{-1} \partial_{\mu} f^{c d}(\operatorname{Re} f)_{d b}^{-1}(D+\bar{D})^{b}\right|^{2}}{\left[T+\bar{T}-\frac{1}{2}(D+\bar{D})^{a}(\operatorname{Re} f)_{a b}^{-1}(D+\bar{D})^{b}\right]^{2}} \\
& -\frac{\left(\partial_{\mu} D^{a}-\frac{1}{2} \partial_{\mu} f^{a c}(\operatorname{Re} f)_{c d}^{-1}(D+\bar{D})^{d}\right)(\operatorname{Re} f)_{a b}^{-1}\left(\partial^{\mu} \bar{D}^{b}-\frac{1}{2} \partial^{\mu} \bar{f} b c(\operatorname{Re} f)_{c d}^{-1}(D+\bar{D})^{d}\right)}{\left[T+\bar{T}-\frac{1}{2}(D+\bar{D})^{a}(\operatorname{Re} f)_{a b}^{-1}(D+\bar{D})^{b}\right]} .
\end{aligned}
$$

With this form of the Lagrangian one verifies eqs. (11), (12).

For completness let us also give the three-dimensional Lagrangian of the heterotic string in the string frame

$$
\begin{aligned}
\mathcal{L}_{\mathrm{str}}^{(3)}= & \lambda_{3}^{-2}\left(\frac{1}{2} R_{\mathrm{str}}^{(3)}-G_{\bar{\imath} j}^{(4)} \partial_{\mu} \bar{\phi}^{\bar{\imath}} \partial^{\mu} \phi^{j}+\frac{2}{\lambda_{3}^{2}} \partial_{\mu} \lambda_{3} \partial^{\mu} \lambda_{3}-\frac{1}{2 r_{s}^{2}} \partial_{\mu} r_{s} \partial^{\mu} r_{s}+\frac{r_{s}^{2}}{4} H_{\mu} H^{\mu}\right. \\
& \left.-\frac{1}{2 r_{s}^{2}} \partial_{\mu} \zeta^{a} \partial^{\mu} \zeta^{a}+\frac{1}{2}\left(F_{\mu}^{a}+\zeta^{a} H_{\mu}\right)^{2}\right)-\frac{1}{4} \lambda_{3}^{2} r_{s}^{2} \partial_{\mu} a \partial^{\mu} a+a \partial_{\mu} \zeta^{a}\left(F^{a \mu}+\zeta^{a} H^{\mu}\right) .
\end{aligned}
$$

The fact, that we do not get an overall factor $\lambda_{3}^{-2}$ is an artefact of the dualisation of the antisymmetric tensor in $D=4$. We see that the perturbation series is governed by the three-dimensional dilaton (18) which also determines the three-dimensional gauge couplings. Notice however, that the gauge coupling for the Kaluza-Klein vector $B_{\mu}$ also depends on the fields $r_{s}$ and $\zeta^{a}$. Dualizing the vectors again yields

$$
\begin{aligned}
\mathcal{L}_{\mathrm{str}}^{(3)}= & \lambda_{3}^{-2}\left(\frac{1}{2} R_{\mathrm{str}}^{(3)}-G_{\bar{\imath} j}^{(4)} \partial_{\mu} \bar{\phi}^{\bar{\imath}} \partial^{\mu} \phi^{j}+\frac{2}{\lambda_{3}^{2}} \partial_{\mu} \lambda_{3} \partial^{\mu} \lambda_{3}-\frac{1}{2 r_{s}^{2}} \partial_{\mu} r_{s} \partial^{\mu} r_{s}-\frac{1}{2 r_{s}^{2}} \partial_{\mu} \zeta^{a} \partial^{\mu} \zeta^{a}\right) \\
& -\lambda_{3}^{2}\left(r_{s}^{2} \partial_{\mu} a \partial^{\mu} a+\frac{1}{4 r_{s}^{2}}\left(\partial_{\mu} b+\zeta^{a} \stackrel{\leftrightarrow}{\partial}_{\mu} C^{a}\right)^{2}+\frac{1}{2}\left(\partial_{\mu} C^{a}-a \partial_{\mu} \zeta^{a}\right)^{2}\right)
\end{aligned}
$$

\footnotetext{
${ }^{19}$ It is essential for this to work that $f_{a b}$ depends holomorphically on the moduli fields, ensuring the identity $\partial_{\Phi^{I}} f_{a b}=2 \partial_{\Phi^{I}}(\operatorname{Re} f)_{a b}$.

${ }^{20}$ We have used the tree level form of the gauge kinetic functions (4) in this formula and inserted $\Phi^{I}=\left(S, \phi^{i}\right)$
} 


\section{Dimensional reduction of $D=11$ supergravity on Calabi-Yau fourfolds}

In this appendix we present some of the technical details connected to the dimensional reduction of the 11-dimensional supergravity Lagrangian (22) on Calabi-Yau fourfolds. Starting from the Ansatz (27) one considers infinitesimally small deformations of the metric, (29) and (30), which preserve the Calabi-Yau condition. These metric deformations correspond to variations of the Kähler class and the complex structure of the Calabi-Yau metric. To lowest order they can be deduced as solutions of the Lichnerowicz equations, which are derived by linearizing the equations for Ricci-flatness of the internal metric, i.e. $R_{a b}^{(8)}(\hat{g}+\delta g)=0$. Solving these equations shows that the metric deformations can (to lowest order) be expanded like in (29) and (30) [67. The expansions are sufficient as we are only interested in the leading terms of the Kaluza-Klein reduced Lagrangian. The only non-vanishing Christoffel symbols apart from $\Gamma_{\mu \nu}^{\rho}$ and the ones with only internal indices are (in complex coordinates)

$$
\begin{aligned}
\Gamma_{\mu i}^{j} & =-\frac{i}{2} g^{\bar{k} j} \partial_{\mu} M^{A} e_{i \bar{k}}^{A}+\frac{1}{2} g^{j k} \partial_{\mu} \bar{Z}^{\bar{\alpha}} b_{k i}^{\bar{\alpha}} \\
\Gamma_{\mu i}^{\bar{\jmath}} & =\frac{1}{2} g^{\bar{\jmath} k} \partial_{\mu} \bar{Z}^{\bar{\alpha}} b_{k i}^{\bar{\alpha}}-\left(\frac{i}{2} g^{\bar{k} \bar{\jmath}} \partial_{\mu} M^{A} e_{i \bar{k}}^{A}\right) \\
\Gamma_{\mu \bar{\jmath}}^{i} & =\frac{1}{2} g^{i \bar{k}} \partial_{\mu} Z^{\alpha} b_{\bar{k} \bar{\jmath}}^{\alpha}-\left(\frac{i}{2} g^{i k} \partial_{\mu} M^{A} e_{k \bar{\jmath}}^{A}\right)=\overline{\Gamma_{\mu j}^{\bar{\alpha}}} \\
\Gamma_{\mu \bar{\jmath}}^{\bar{\imath}} & =-\frac{i}{2} g^{\bar{\imath} k} \partial_{\mu} M^{A} e_{k \bar{\jmath}}^{A}+\frac{1}{2} g^{\bar{\imath} \bar{k}} \partial_{\mu} Z^{\alpha} b_{\bar{k} \bar{\jmath}}^{\alpha}=\overline{\Gamma_{\mu j}^{i}} \\
\Gamma_{i j}^{\mu} & =-\frac{1}{2} g^{\mu \nu} \partial_{\nu} \bar{Z}^{\bar{\alpha}} b_{i j}^{\bar{\alpha}}, \\
\Gamma_{i \bar{\jmath}}^{\mu} & =\frac{i}{2} g^{\mu \nu} \partial_{\nu} M^{A} e_{i \bar{\jmath}}^{A}=\Gamma_{\bar{\jmath} i}^{\mu}, \\
\Gamma_{\bar{\imath} \bar{\jmath}}^{\mu} & =-\frac{1}{2} g^{\mu \nu} \partial_{\nu} Z^{\alpha} b_{\bar{\imath} \bar{\jmath}}^{\alpha}=\overline{\Gamma_{i j}^{\mu}} .
\end{aligned}
$$

The terms in brackets do not contribute to leading order in the expansion of $R^{(11)}$. The 11-dimensional curvature scalar splits into

$$
R^{(11)}=2 g^{i \bar{\jmath}} R_{i \bar{\jmath}}^{(11)}+g^{i j} R_{i j}^{(11)}+g^{\bar{\imath} \bar{\jmath}} R_{\bar{\imath} \bar{\jmath}}^{(11)}+g^{\mu \nu} R_{\mu \nu}^{(11)},
$$

where we have used the fact, that $g^{i \bar{\jmath}} R_{i \bar{\jmath}}^{(11)}$ is real and therefore equal to $g^{\bar{\imath} j} R_{\bar{\imath} j}^{(11)}$. We have

$$
\begin{aligned}
R_{i \bar{\jmath}}^{(11)} & =R^{(11) k}{ }_{i k \bar{\jmath}}+R^{(11) \bar{k}}{ }_{i \bar{k} \bar{\jmath}}+R^{(11) \mu}{ }_{i \mu \bar{\jmath}}, \\
R_{\mu \nu}^{(11)} & =R_{\mu k \nu}^{(11) k}+R_{\mu \bar{k} \nu}^{(11) \bar{k}}+R_{\mu \lambda \nu}^{(11) \lambda}
\end{aligned}
$$

and similar equations for $R_{i j}^{(11)}$ and $R_{\bar{\imath} \bar{\jmath}}^{(11)}$. Furthermore certain components of the 11dimensional curvature tensor are related to components of the (Ricci-flat) internal and the external curvature tensors:

$$
\begin{aligned}
R_{\mu \lambda \nu}^{(11) \lambda} & =R_{\mu \lambda \nu}^{(3) \lambda}, \\
R_{i k \bar{\jmath}}^{(11) k} & =R^{(8) k}{ }_{i k \bar{\jmath}}+\Gamma_{i \bar{\jmath}}^{\mu} \Gamma_{\mu k}^{k}-\Gamma_{i k}^{\mu} \Gamma_{\mu \bar{\jmath}}^{k}, \quad \text { etc. . }
\end{aligned}
$$


Using these relations and the Christoffel symbols of (C.21) one derives to lowest order in the moduli

$$
\begin{gathered}
\frac{1}{2} \int d^{11} x \sqrt{-g^{(11)}} R^{(11)}=\int d^{3} x \sqrt{-g^{(3)}} \int d^{8} \xi \sqrt{\hat{g}}\left(\frac{1}{2} R^{(3)}-\frac{1}{4} \partial_{\mu} Z^{\alpha} \partial^{\mu} \bar{Z}^{\bar{\beta}} b_{\bar{\jmath} \bar{m}}^{\alpha} \bar{b}_{i k}^{\bar{\beta}} \hat{g}^{i \bar{\jmath}} \hat{g}^{k \bar{m}}\right. \\
\left.-\frac{1}{2} \partial_{\mu} M^{A} \partial^{\mu} M^{B} e_{i \bar{\jmath}}^{A} e_{k \bar{m}}^{B} \hat{g}^{i \bar{\jmath}} \hat{g}^{k \bar{m}}+\frac{1}{4} \partial_{\mu} M^{A} \partial^{\mu} M^{B} e_{i \bar{\jmath}}^{A} e_{k \bar{m}}^{B} \hat{g}^{i \bar{m}} \hat{g}^{k \bar{\jmath}}\right),
\end{gathered}
$$

where a total derivative has been neglected.

For the reduction of the remaining terms in (22) we have to expand the three-form $A_{3}$ in terms of the (1,1)-forms $e^{A}$ and $(2,1)$-forms $\Psi^{I}$ :

$$
A_{3}=A_{\mu}^{A} d x^{\mu} \wedge e^{A}+N^{I} \Psi^{I}+\bar{N}^{\bar{J}} \bar{\Psi}^{\bar{J}}
$$

Using (42) one derives

$$
F_{4}=\frac{1}{2} F_{\mu \nu}^{A} d x^{\mu} \wedge d x^{\nu} \wedge e^{A}+D_{\mu} N^{I} d x^{\mu} \wedge \Psi^{I}+D_{\mu} \bar{N}^{\bar{J}} d x^{\mu} \wedge \bar{\Psi}^{\bar{J}}
$$

where we abbreviated

$$
D_{\mu} N^{I}=\partial_{\mu} N^{I}+N^{K} \sigma_{\alpha K I} \partial_{\mu} Z^{\alpha}+\bar{N}^{\bar{L}} \bar{\tau}_{\bar{\beta} \bar{L} I} \partial_{\mu} \bar{Z}^{\bar{\beta}}, \quad D_{\mu} \bar{N}^{\bar{J}}=\overline{D_{\mu} N^{J}} .
$$

Inserting (C.26) and (C.27) into (22) one derives to lowest order

$$
\begin{aligned}
& -\frac{1}{4} \int d^{11} x \sqrt{-g^{(11)}}\left|F_{4}\right|^{2}= \\
& \quad-\frac{1}{8} \int d^{3} x \sqrt{-g^{(3)}} \int d^{8} \xi \sqrt{\hat{g}}\left(F_{\mu \nu}^{A} F^{B \mu \nu} e_{i \bar{\jmath}}^{A} e_{k \bar{m}}^{B} \hat{g}^{i \bar{m}} \hat{g}^{k \bar{\jmath}}+D_{\mu} N^{I} D^{\mu} \bar{N}^{\bar{J}} \Psi_{i j \bar{k}}^{I} \bar{\Psi}_{l \bar{m} \bar{n}}^{\bar{J}} \hat{g}^{i \bar{m}} \hat{g}^{j \bar{n}} \hat{g}^{l \bar{k}}\right)
\end{aligned}
$$

and

$$
\begin{aligned}
& -\frac{1}{12} \int A_{3} \wedge F_{4} \wedge F_{4}= \\
& \quad-\frac{1}{16} \int d^{3} x \sqrt{-g^{(3)}} \epsilon^{\mu \nu \rho} A_{\mu}^{A} D_{\nu} N^{I} D_{\rho} \bar{N}^{\bar{J}} \int d^{8} \xi \sqrt{\hat{g}} \epsilon^{i k l s} \epsilon^{\bar{\jmath} \bar{m} \bar{n} \bar{r}} e_{i \bar{\jmath}}^{A} \Psi_{k l \bar{m}}^{I} \bar{\Psi}_{s \bar{n} \bar{r}}^{\bar{J}}
\end{aligned}
$$

Before we proceed let us define (in close analogy with [58])

$$
\begin{aligned}
\mathcal{V} & \equiv \frac{1}{4 !} \int_{Y_{4}} J \wedge J \wedge J \wedge J \\
\mathcal{V}_{A} & \equiv \frac{1}{4 !} \int_{Y_{4}} e^{A} \wedge J \wedge J \wedge J \\
\mathcal{V}_{A B} & \equiv \frac{1}{4 !} \int_{Y_{4}} e^{A} \wedge e^{B} \wedge J \wedge J \\
& =\frac{1}{12} \int_{Y_{4}} d^{8} \xi \sqrt{g} e_{i \bar{\jmath}}^{A} e_{k \bar{m}}^{B} g^{i \bar{m}} g^{k \bar{\jmath}}-\frac{1}{12} \int_{Y_{4}} d^{8} \xi \sqrt{g} e_{i \bar{\jmath}}^{A} e_{k \bar{m}}^{B} g^{i \bar{\jmath}} g^{k \bar{m}},
\end{aligned}
$$

\footnotetext{
${ }^{21}$ In order to derive this form of the reduced action one has to perform a partial integration and use the relation $d_{A I \bar{J}} \tau_{\alpha K \bar{J}}=d_{A K \bar{J}} \tau_{\alpha I \bar{J}}$, which can be easily derived from $\partial_{Z^{\alpha}} \int e^{A} \wedge \Psi^{I} \wedge \Psi^{K}=0$.
} 
where $J$ is the Kähler form defined in eq. (35). With the help of (C.31) one derives (again in close analogy with the threefold case 61])

$$
\begin{aligned}
\star e^{A} & =\frac{2}{3} \frac{\mathcal{V}_{A}}{\mathcal{V}} J \wedge J \wedge J-\frac{1}{2} e^{A} \wedge J \wedge J \\
G_{A B} & =-6 \frac{\mathcal{V}_{A B}}{\mathcal{V}}+8 \frac{\mathcal{V}_{A} \mathcal{V}_{B}}{\mathcal{V}^{2}}=-\frac{1}{2} \partial_{A} \partial_{B} \ln \mathcal{V}
\end{aligned}
$$

and

$$
16 \frac{\mathcal{V}_{A} \mathcal{V}_{B}}{\mathcal{V}^{2}} \partial_{\mu} M^{A} \partial^{\mu} M^{B}=\partial_{\mu} \ln \mathcal{V} \partial^{\mu} \ln \mathcal{V}
$$

The integrals over the internal coordinates in $(\overline{C .25})$, (C.29) and (C.30) can be performed using the definitions (33), (36) and (38).22 With the help of (C.32), (C.33) and performing a Weyl rescaling $g_{\mu \nu}^{(3)} \rightarrow \mathcal{V}^{2} g_{\mu \nu}^{(3)}$ one derives

$$
\begin{aligned}
\mathcal{L}^{(3)}= & \frac{1}{2} R^{(3)}-\frac{1}{2} \partial_{\mu} \ln \mathcal{V} \partial^{\mu} \ln \mathcal{V}-G_{\alpha \bar{\beta}} \partial_{\mu} Z^{\alpha} \partial^{\mu} \bar{Z}^{\bar{\beta}}-\mathcal{V}^{-1} G_{I \bar{J}} D_{\mu} N^{I} D^{\mu} \bar{N}^{\bar{J}} \\
& -\frac{1}{2} G_{A B} \partial_{\mu} M^{A} \partial^{\mu} M^{B}-\frac{1}{4} \mathcal{V}^{2} G_{A B} F_{\mu \nu}^{A} F^{B \mu \nu}-\frac{1}{4} \epsilon^{\mu \nu \rho} d_{A I \bar{J}} A_{\mu}^{A} D_{\nu} N^{I} D_{\rho} \bar{N}^{\bar{J}} .
\end{aligned}
$$

The three-dimensional vectors are dualised to scalars by adding the Lagrange multipliers $P^{A}$

$$
-F_{\mu}^{A} \partial^{\mu} P^{A}, \quad F^{A \rho}=\frac{1}{2} \epsilon^{\rho \mu \nu} F_{\mu \nu}^{A}
$$

and eliminating the fields $F_{\mu}^{A}$ via their equations of motion. The result is

$$
\begin{aligned}
\mathcal{L}^{(3)}= & \frac{1}{2} R^{(3)}-G_{\alpha \bar{\beta}} \partial_{\mu} Z^{\alpha} \partial^{\mu} \bar{Z}^{\bar{\beta}}-\mathcal{V}^{-1} G_{I \bar{J}} D_{\mu} N^{I} D^{\mu} \bar{N}^{\bar{J}} \\
& -\frac{1}{2} \partial_{\mu} \ln \mathcal{V} \partial^{\mu} \ln \mathcal{V}-\frac{1}{2} G_{A B} \partial_{\mu} M^{A} \partial^{\mu} M^{B} \\
& -\frac{1}{2 \mathcal{V}^{2}}\left(\partial_{\mu} P^{A}+\frac{1}{8} d_{A K \bar{L}}\left(N^{K} D_{\mu} \bar{N}^{\bar{L}}-D_{\mu} N^{K} \bar{N}^{\bar{L}}\right)\right) \\
& G_{A B}^{-1}\left(\partial^{\mu} P^{B}+\frac{1}{8} d_{B I \bar{J}}\left(N^{I} D^{\mu} \bar{N}^{\bar{J}}-D^{\mu} N^{I} \bar{N}^{\bar{J}}\right)\right) .
\end{aligned}
$$

In order to find the Kähler potential for the scalars in (C.36) one introduces the coordinates (46), (47). With this redefinition the derivatives (C.28) take the form

$$
\begin{aligned}
D_{\mu} \bar{N}^{\bar{J}} & =\hat{G}_{\bar{J} I}^{-1}\left[\partial_{\mu} \hat{N}^{I}+\partial_{\mu} Z^{\alpha}\left(\hat{G}_{\bar{M} K}^{-1} \overline{\hat{N}}^{\bar{M}} \tau_{\alpha K \bar{L}} \hat{G}_{I \bar{L}}-\sigma_{\alpha I K} \hat{N}^{K}\right)\right] \equiv \hat{G}_{\bar{J} I}^{-1} D_{\mu} \hat{N}^{I}, \\
D_{\mu} N^{I} & =\hat{G}_{\bar{J} I}^{-1}\left[\partial_{\mu} \overline{\hat{N}}^{\bar{J}}+\partial_{\mu} \bar{Z}^{\bar{\beta}}\left(\hat{G}_{\bar{M} K}^{-1} \hat{N}^{K} \bar{\tau}_{\bar{\beta} \bar{M} L} \hat{G}_{L \bar{J}}-\bar{\sigma}_{\bar{\beta} \bar{J} \bar{M}} \overline{\hat{N}}^{\bar{M}}\right)\right] \equiv \hat{G}_{\bar{J} I}^{-1} D_{\mu} \overline{\hat{N}}^{\bar{J}} .
\end{aligned}
$$

\footnotetext{
${ }^{22}$ Strictly speaking one has to replace the Calabi-Yau metric in these definitions by its background value. This is related to the fact that we perform the Kaluza-Klein reduction to first non-trivial order around a fixed but arbitray point in the moduli space of metrics. This procedure results in couplings in the effective Lagrangian which depend on the arbitrary background values. Using field theoretical reasoning this can be promoted to full non-linear $\sigma$-model type couplings.
} 
The Lagrangian (C.36) becomes

$$
\begin{aligned}
\mathcal{L}^{(3)}= & \frac{1}{2} R^{(3)}-G_{\alpha \bar{\beta}} \partial_{\mu} Z^{\alpha} \partial^{\mu} \bar{Z}^{\bar{\beta}}-\mathcal{V}^{-1} G_{I \bar{J}} \hat{G}_{\bar{L} I}^{-1} \hat{G}_{\bar{J} M}^{-1} D_{\mu} \overline{\hat{N}}^{\bar{L}} D^{\mu} \hat{N}^{M} \\
& -\frac{1}{2} \partial_{\mu} \ln \mathcal{V} \partial^{\mu} \ln \mathcal{V}-\frac{1}{2} G_{A B} \partial_{\mu} M^{A} \partial^{\mu} M^{B} \\
& -\frac{1}{2 \mathcal{V}^{2}}\left(\partial_{\mu} P^{A}+\frac{1}{8} d_{A K \bar{L}} \hat{G}_{\bar{N} K}^{-1} \hat{G}_{\bar{L} M}^{-1}\left(\overline{\hat{N}}^{\bar{N}} D_{\mu} \hat{N}^{M}-D_{\mu} \overline{\hat{N}^{N}} \hat{N}^{M}\right)\right) \\
& G_{A B}^{-1}\left(\partial^{\mu} P^{B}+\frac{1}{8} d_{B I \bar{J}} \hat{G}_{\bar{P} I}^{-1} \hat{G}_{\bar{J} Q}^{-1}\left(\overline{\hat{N}}^{\bar{P}} D^{\mu} \hat{N}^{Q}-D^{\mu} \overline{\hat{N}}^{\bar{P}} \hat{N}^{Q}\right)\right) .
\end{aligned}
$$

Using (46)-(50) one verifies that $\mathcal{L}^{(3)}$ given in (C.38) coincides with the Lagrangian of (45).

\section{References}

[1] For a review see, for example,

E. Kiritsis, "Introduction to non-perturbative String Theory", hep-th/9708130;

B. de Wit and J. Louis, "Supersymmetry and Dualities in Various Dimensions", hep-th/9801132;

A. Sen, "An Introduction to Non-perturbative String Theory", hep-th/9802051;

M. Haack, B. Körs and D. Lüst, "Recent Developments in String Theory: From Perturbative Dualities to M-Theory", hep-th/9904033.

[2] For a review see, for example,

L.E. Ibanez, "The second string (phenomenology) revolution", hep-ph/9911499, and references therein.

[3] C. Vafa, "Evidence for F Theory", Nucl. Phys. B469 (1996) 403, hep-th/9602022.

[4] D. Morrison and C. Vafa, "Compactifications of F-Theory on Calabi-Yau Threefolds - I", Nucl. Phys. B473 (1996) 74, hep-th/9602114.

[5] D. Morrison and C. Vafa, "Compactifications of F-Theory on Calabi-Yau Threefolds - II", Nucl. Phys. B476 (1996) 437, hep-th/9603161.

[6] E. Witten, (1996) "Nonperturbative Superpotentials in String Theory", Nucl. Phys. B474 (1996) 343, hep-th/9604030.

[7] K. Becker and M. Becker, "M-Theory on Eight-Manifolds", Nucl. Phys. B477 (1996) 155, hep-th/9605053.

[8] S. Sethi, C. Vafa and E. Witten, "Constraints on Low Dimensional String Compactifications", Nucl. Phys. B480 (1996) 213, hep-th/9606122.

[9] I. Brunner and R. Schimmrigk, "F-Theory on Calabi-Yau Fourfolds", Phys. Lett. B387 (1996) 750, hep-th/9606148. 
[10] R. Gopakumar and S. Mukhi, "Orbifold and Orientifold Compactifications of FTheory and M-Theory to Six and Four Dimensions", Nucl. Phys. B479 (1996) 260, hep-th/9607057.

[11] R. Donagi, A. Grassi and E. Witten, "A Nonperturbative Superpotential With E(8) Symmetry", Mod. Phys. Lett. A11 (1996) 2199, hep-th/9607091.

[12] E. Witten, "On Flux Quantization in M-Theory and the Effective Action", J. Geom. Phys. 22 (1997) 1, hep-th/9609122.

[13] P. Mayr, "Mirror Symmetry, N=1 Superpotentials and Tensionless Strings on Calabi-Yau Fourfolds", Nucl. Phys. B494 (1997) 489, hep-th/9610162.

[14] I. Brunner, M. Lynker and R. Schimmrigk, "Unification of M-and F-Theory CalabiYau Fourfold Vacua", Nucl. Phys. B498 (1997) 156, hep-th/9610195.

[15] S. Katz and C. Vafa, "Geometric Engineering of N=1 Quantum Field Theories", Nucl. Phys. B497 (1997) 196, hep-th/9611090.

[16] M. Bershadsky, A. Johansen, T. Pantev, V. Sadov and C. Vafa, "F-Theory, Geometric Engineering and N=1 Dualities", Nucl. Phys. B505 (1997) 153, hep-th/9612052.

[17] K. dasgupta and S. Mukhi, "A Note on Low-Dimensional String Compactifications", Phys. Lett. B398 (1997) 285, hep-th/9612188.

[18] A. Klemm, B. Lian, S.S. Roan and S.T. Yau, "Calabi-Yau Fourfolds for M- and F-Theory Compactifications", Nucl. Phys. B518 (1998) 515, hep-th/9701023.

[19] K. Mohri, "F-Theory Vacua in Four Dimensions and Toric Threefolds", Int. J. Mod. Phys. A14 (1999) 845, hep-th/9701147.

[20] R. Friedman, J. Morgan and E. Witten, "Vector Bundles and F-Theory", Commun. Math. Phys. 187 (1997) 679, hep-th/9701162.

[21] M. Bershadsky, A. Johansen, T. Pantev and V. Sadov, "On Four-Dimensional Compactifications of F-Theory", Nucl. Phys. B505 (1997) 165, hep-th/9701165.

[22] M. Kreuzer and H. Skarke, "Calabi-Yau 4-Folds and Toric Fibrations", J. Geom. Phys. 26 (1998) 272, hep-th/9701175.

[23] G. Curio and D. Lüst, "A Class of N=1 Dual String Pairs and its Modular Superpotential", Int. J. Mod. Phys. A12 (1997) 5847, hep-th/9703007.

[24] I. Brunner, M. Lynker and R. Schimmrigk, "Dualities and Phase Transitions for Calabi-Yau Threefolds and Fourfolds", Nucl. Phys. Proc. Suppl. 56B (1997) 120, hep-th/9703182.

[25] B. Andreas, G. Curio and D. Lüst, " $\mathrm{N}=1$ Dual String Pairs and Their Massless Spectra", Nucl. Phys. B507 (1997) 175, hep-th/9705174.

[26] B. Andreas and G. Curio, "Three-Branes and Five-Branes in N=1 Dual String Pairs", Phys. Lett. B417 (1998) 41, hep-th/9706093. 
[27] V. Kaplunovsky and J. Louis, "Phenomenological Aspects of F-Theory", Phys. Lett. B417 (1998) 45, hep-th/9708049.

[28] D. Lüst, "N=1 Dual String Pairs and their Modular Superpotentials", Nucl. Phys. Proc. Suppl. 68 (1998) 66, hep-th/9709222.

[29] M. Bershadsky, T.M. Chiang, B.R. Greene, A. Johansen and C.I. Lazaroiu, "FTheory and Linear Sigma Models", Nucl. Phys. B527 (1998) 531, hep-th/9712023.

[30] G. Curio and R. Donagi, "Moduli in N=1 Heterotic/F-Theory Duality", Nucl. Phys. B518 (1998) 603, hep-th/9801057.

[31] G. Curio, "Chiral Matter and Transitions in Heterotic string Models", Phys. Lett. B435 (1998) 39, hep-th/9803224.

[32] B. Andreas, "N=1 Heterotic/F-Theory Duality", Fortsch. Phys. 47 (1999) 587, hepth/9808159.

[33] P. Berglund and P. Mayr, "Heterotic String/F-Theory Duality from Mirror Symmetry", Adv. Theor. Math. Phys. 2 (1999) 1307, hep-th/9811217.

[34] G. Rajesh, "Toric Geometry and F-Theory/Heterotic Duality in Four Dimensions", JHEP 9812:018 (1998), hep-th/9811240.

[35] M. Lynker, R. Schimmrigk and A. Wisskirchen, "Landau-Ginzburg Vacua of String, M- and F-Theory at c=12", Nucl. Phys. B550 (1999) 123, hep-th/9812195.

[36] D.-E. Diaconescu and G. Rajesh, "Geometrical Aspects of Fivebranes in Heterotic/F-Theory Duality in Four Dimensions", JHEP 9906:002 (1999), hep-th/9903104.

[37] P. Mayr, "N=1 Heterotic String Vacua from Mirror Symmetry", hep-th/9904115.

[38] S. Gukov, C. Vafa and E. Witten, "CFT's from Calabi-Yau Four-Folds", hepth/9906070.

[39] K. Dasgupta, G. Rajesh and S. Sethi, "M-Theory, Orientifolds and G-Flux", JHEP 9908:023 (1999), hep-th/9908088.

[40] B. Andreas and G. Curio, "On Discrete Twist and Four-Flux in N=1 Heterotic/FTheory Compactifications", hep-th/9908193.

[41] B. Andreas and G. Curio, "Horizontal and Vertical Five-Branes in Heterotic/FTheory Duality", hep-th/9912025.

[42] B. Zumino, "Supersymmetry and Kähler Manifolds", Phys. Lett. 87B (1979) 203.

[43] B. de Wit, H. Nicolai and A.K. Tollstén, "Locally Supersymmetric $D=3$ Non-Linear Sigma Models", Nucl. Phys. B392 (1993) 3, hep-th/9208074.

[44] A. Klemm, W. Lerche and P. Mayr, "K3-Fibrations and Heterotic-Type II String Duality", Phys. Lett. B357 (1995) 313, hep-th/9506112. 
[45] P. Aspinwall and J. Louis, "On the Ubiquity of K3 Fibrations in String Duality", Phys. Lett. B369 (1996) 233, hep-th/9510234.

[46] M. Haack and J. Louis, "Aspects of Heterotic/M-Theory Duality in $D=3$ ", hepth/9908067.

[47] For a review see, for example, K. Förger and J. Louis, "Holomorphic Couplings in String Theory", Nucl. Phys. Proc. Suppl. 55B (1997) 33, hep-th/9611184.

[48] S. Ferrara and A. Strominger, "N=2 Space-Time Supersymmetry and Calabi-Yau Moduli Space", in Proceedings of "Strings 89", eds. R. Arnowitt, R. Bryan, M. Duff, D. Nanopoulos and C. Pope, World Scientific, Singapore, 1990.

[49] P. Candelas and X.C. de la Ossa, "Moduli Space of Calabi-Yau Manifolds", Nucl. Phys. B355 (1991) 455.

[50] A. Strominger, "Special Geometry", Commun. Math. Phys. 133 (1990) 163.

[51] P. Candelas, X. de la Ossa, P. Green and L. Parkes, "An Exactly Soluble Superconformal Theory from a Mirror Pair of Calabi-Yau Manifolds", Nucl. Phys. B359 (1991) 21.

[52] S. Ferrara and S. Sabharwal, "Quaternionic Manifolds for Type II Superstring Vacua of Calabi-Yau Spaces", Nucl. Phys. B332 (1990) 317.

[53] E. Witten, "String Theory Dynamics in Various Dimensions", Nucl. Phys. B443 (1995) 85, hep-th/9503124.

[54] E. Cremmer, B. Julia and J. Scherk, "Supergravity Theory in Eleven-Dimensions", Phys. Lett. B76 (1978) 409.

[55] C. Vafa and E. Witten, "A One Loop Test of String Duality", Nucl. Phys. B447 (1995) 261, hep-th/9505053.

[56] M.J. Duff, J.T. Liu and R. Minasian, "Eleven-Dimensional Origin of String-String Duality: A One Loop Test", Nucl. Phys. B452 (1995) 261, hep-th/9506126.

[57] A. Strominger, "Superstrings with Torsion", Nucl. Phys. B274 (1986) 253.

[58] M. Bodner, A.C. Cadavid and S. Ferrara, "(2,2) Vacuum Configurations for Type IIA Superstrings: N=2 Supergravity Lagrangians and Algebraic Geometry", Class. Quantum Grav. 8 (1991) 789.

[59] A.C. Cadavid, A. Ceresole, R. D'Auria and S. Ferrara, "Eleven-Dimensional Supergravity Compactified on Calabi-Yau Threefolds", Phys. Lett. B357 (1995) 76 , hep-th/9506144.

[60] I. Antoniadis, S. Ferrara and T.R. Taylor, "N=2 Heterotic Superstring and its Dual Theory in Five Dimensions", Nucl. Phys. B460 (1996) 489, hep-th/9511108.

[61] A. Strominger, "Yukawa Couplings in Superstring Compactification", Phys. Rev. Lett. 55 (1985) 2547. 
[62] C. Vafa and E. Witten, "Dual String Pairs with $N=1$ and $N=2$ Supersymmetry in Four Dimensions", Nucl. Phys. Proc. Suppl. 46 (1996) 225, hep-th/9507050.

[63] P. Aspinwall, "K3 Surfaces and String Duality", hep-th 9611137.

[64] J. Louis, J. Sonnenschein, S. Theisen and S. Yankielowicz, "Non-Perturbative Properties of Heterotic String Vacua Compactified on K3×T ${ }^{2}$ ", Nucl. Phys. B480 (1996) 185, hep-th/9606049.

[65] M. Haack and J. Louis, in preparation.

[66] C.W. Misner, K.S. Thorne and J.A. Wheeler, "Gravitation", San Francisco: Free$\operatorname{man}(1973)$.

[67] P. Candelas, "Yukawa Couplings Between (2,1)-Forms", Nucl. Phys. B298 (1988) 458. 